\title{
A general composite iterative method for generalized mixed equilibrium problems, variational inequality problems and optimization problems
}

Jong Soo Jung

Correspondence: jungjs@mail. donga.ac.kr

Department of Mathematics, Dong-A University, Busan, 604-714, Korea

\begin{abstract}
In this article, we introduce a new general composite iterative scheme for finding a common element of the set of solutions of a generalized mixed equilibrium problem, the set of fixed points of an infinite family of nonexpansive mappings and the set of solutions of a variational inequality problem for an inverse-strongly monotone mapping in Hilbert spaces. It is shown that the sequence generated by the proposed iterative scheme converges strongly to a common element of the above three sets under suitable control conditions, which solves a certain optimization problem. The results of this article substantially improve, develop, and complement the previous well-known results in this area.

2010 Mathematics Subject Classifications: 49J30; 49J40; 47H09; 47H10; 47J20; 47J25; 47J05; 49M05.
\end{abstract}

Keywords: generalized mixed equilibrium problem, fixed point, nonexpansive mapping; inverse-strongly monotone mapping, variational inequality; optimization problem, metric projection, strongly positive bounded linear operator

\section{Introduction}

Let $H$ be a real Hilbert space with inner product $\langle\cdot, \cdot\rangle$ and induced norm $\|\cdot\|$. Let $C$ be a nonempty closed convex subset of $H$ and $S: C \rightarrow C$ be a self-mapping on $C$. Let us denote by $F(S)$ the set of fixed points of $S$ and by $P_{C}$ the metric projection of $H$ onto $C$.

Let $B: C \rightarrow H$ be a nonlinear mapping and $\phi: C \rightarrow \mathbb{R}$ be a function, and $\Theta$ be a bifunction of $C \times C$ into $\mathbb{R}$, where $\mathbb{R}$ is the set of real numbers.

Then, we consider the following generalized mixed equilibrium problem of finding $x \in C$ such that

$$
\Theta(x, y)+\langle B x, y-x\rangle+\varphi(y)-\varphi(x) \geq 0, \quad \forall y \in C,
$$

which was recently introduced by Peng and Yao [1]. The set of solutions of the problem (1.1) is denoted by $\operatorname{GMEP}(\Theta, \phi, B)$. Here, some special cases of the problem (1.1) are stated as follows: 
If $B=0$, then the problem (1.1) reduces the following mixed equilibrium problem of finding $x \in C$ such that

$$
\Theta(x, y)+\varphi(y)-\varphi(x) \geq 0, \quad \forall y \in C,
$$

which was studied by Ceng and Yao [2] (see also [3]). The set of solutions of the problem (1.2) is denoted by $M E P(\Theta, \phi)$.

If $\phi=0$ and $B=0$, then the problem (1.1) reduces the following equilibrium problem of finding $x \in C$ such that

$$
\Theta(x, y) \geq 0, \quad \forall y \in C .
$$

The set of solutions of the problem (1.3) is denoted by $E P(\Theta)$.

If $\phi=0$ and $\Theta(x, y)=0$ for all $x, y \in C$, then the problem (1.1) reduces the following variational inequality problem of finding $x \in C$ such that

$$
\langle B x, y-x\rangle \geq 0, \quad \forall y \in C .
$$

The set of solutions of the problem (1.4) is denoted by $V I(C, B)$.

The problem (1.1) is very general in the sense that it includes, as special cases, fixed point problems, optimization problems, variational inequality problems, minmax problems, Nash equilibrium problems in noncooperative games, and others; see [2,4-6].

Recently, in order to study the problem (1.3) coupled with the fixed point problem, many authors have introduced some iterative schemes for finding a common element of the set of the solutions of the problem (1.3) and the set of fixed points of a countable family of nonexpansive mappings; see [7-16] and the references therein.

In 2008, Su et al. [17] gave an iterative scheme for the problem (1.3), the problem (1.4) for an inverse-strongly monotone mapping, and fixed point problems of nonexpansive mappings. In 2009, Yao et al. [18] considered an iterative scheme for the problem (1.2), the problem (1.4) for a Lipschitz and relaxed-cocoercive mapping and fixed point problems of nonexpansive mappings, and in 2008, Peng and Yao [1] studied an iterative scheme for the problem (1.1), the problem (1.4) for a monotone, and Lipschitz continuous mapping and fixed point problems of nonexpansive mappings.

In particular, in 2010, Jung [9] introduced the following new composite iterative scheme for finding a common element of the set of solutions of the problem (1.3) and the set of fixed points of a nonexpansive mapping: $x_{1} \in C$ and

$$
\left\{\begin{array}{l}
\Theta\left(u_{n}, y\right)+\frac{1}{r_{n}}\left\langle y-u_{n}, u_{n}-x_{n}\right\rangle \geq 0, \quad \forall y \in C \\
y_{n}=\alpha_{n} f\left(x_{n}\right)+\left(1-\alpha_{n}\right) T u_{n} \\
x_{n+1}=\left(1-\beta_{n}\right) y_{n}+\beta_{n} T y_{n}, \quad n \geq 1
\end{array}\right.
$$

where $T$ is a nonexpansive mapping, $f$ is a contraction with constant $k \in(0,1),\left\{\alpha_{n}\right\}$, $\left\{\beta_{n}\right\} \subset[0,1]$, and $\left\{r_{n}\right\} \subset(0, \infty)$. He showed that the sequences $\left\{x_{n}\right\}$ and $\left\{u_{n}\right\}$ generated by (1.5) converge strongly to a point in $F(T) \cap E P(\Theta)$ under suitable conditions.

On the other hand, the following optimization problem has been studied extensively by many authors:

$$
\min _{x \in \Omega} \frac{\mu}{2}\langle A x, x\rangle+\frac{1}{2}\|x-u\|^{2}-h(x),
$$


where $\Omega=\bigcap_{n=1}^{\infty} C_{n}, C_{1}, C_{2}, \cdots$ are infinitely many closed convex subsets of $H$ such that $\bigcap_{n=1}^{\infty} C_{n} \neq \emptyset, u \in H, \mu \geq 0$ is a real number, $A$ is a strongly positive bounded linear operator on $H$ (i.e., there is a constant $\bar{\gamma}>0$ such that $\langle A x, x\rangle \geq \bar{\gamma}\|x\|^{2}, \forall x \in H$ ) and $h$ is a potential function for $\gamma f$ (i.e., $h^{\prime}(x)=\gamma f(x)$ for all $x \in H$ ). For this kind of optimization problems, see, for example, Bauschke and Borwein [19], Combettes [20], Deutsch and Yamada [21], Jung [22], and Xu [23] when $\Omega=\bigcap_{i=1}^{N} C_{i}$; and $h(x)=\langle x, b\rangle$ for a given point $b$ in $H$.

In 2009, Yao et al. [3] considered the following iterative scheme for the problem (1.2) and optimization problems:

$$
\left\{\begin{array}{l}
\Theta\left(y_{n}, \gamma\right)+\varphi(y)-\varphi\left(y_{n}\right)+\frac{1}{r}\left\langle K^{\prime}\left(y_{n}\right)-K^{\prime}\left(x_{n}\right), y-y_{n}\right\rangle \geq 0, \quad \forall y \in H, \\
x_{n+1}=\alpha_{n}\left(u+\gamma f\left(x_{n}\right)\right)+\beta_{n} x_{n}+\left(\left(1-\beta_{n}\right) I-\alpha_{n}(I+\mu A)\right) W_{n} y_{n}, \quad n \geq 1,
\end{array}\right.
$$

where $u \in H ;\left\{\alpha_{n}\right\}$ and $\left\{\beta_{n}\right\}$ are two sequences in $(0,1), \mu>0, r>0, \gamma>0 ; K^{\prime}(x)$ is the Fréchet derivative of a functional $K: H \rightarrow \mathbb{R}$ at $x$; and $W_{n}$ is the so-called $W$-mapping related to a sequence $\left\{T_{n}\right\}$ of nonexpansive mappings. They showed that under appropriate conditions, the sequences $\left\{x_{n}\right\}$ and $\left\{y_{n}\right\}$ generated by (1.6) converge strongly to a solution of the optimization problem:

$$
\min _{x \in \bigcap_{n=1}^{\infty} F\left(T_{n}\right) \cap M E P(\Theta, \varphi)} \frac{\mu}{2}\langle A x, x\rangle+\frac{1}{2}\|x-u\|^{2}-h(x) .
$$

In 2010, using the method of Yao et al. [3], Jaiboon and Kumam [24] also introduced a general iterative method for finding a common element of the set of solutions of the problem (1.2), the set of fixed points of a sequence $\left\{T_{n}\right\}$ of nonexpansive mappings, and the set of solutions of the problem (1.4) for a $\alpha$-inverse-strongly monotone mapping. We point out that in the main results of $[3,24]$, the condition of the sequentially continuity from the weak topology to the strong topology for the derivative $K^{\prime}$ of the function $K: C \rightarrow \mathbb{R}$ is very strong. Even if $K(x)=\frac{\|x\|^{2}}{2}$, then $K^{\prime}(x)=x$ is not sequentially continuous from the weak topology to the strong topology.

In this article, inspired and motivated by above mentioned results, we introduce a new iterative method for finding a common element of the set of solutions of a generalized mixed equilibrium problem (1.1), the set of fixed points of a countable family of nonexpansive mappings, and the set of solutions of the variational inequality problem (1.4) for an inverse-strongly monotone mapping in a Hilbert space. We show that under suitable conditions, the sequence generated by the proposed iterative scheme converges strongly to a common element of the above three sets, which is a solution of a certain optimization problem. The results of this article can be viewed as an improvement and complement of the recent results in this direction.

\section{Preliminaries and lemmas}

Let $H$ be a real Hilbert space, and let $C$ be a nonempty closed convex subset of $H$. In the following, we write $x_{n} \rightarrow x$ to indicate that the sequence $\left\{x_{n}\right\}$ converges weakly to $x . x_{n} \rightarrow x$ implies that $\left\{x_{n}\right\}$ converges strongly to $x$. 
First, we know that a mapping $f: C \rightarrow C$ is a contraction on $C$ if there exists a constant $k \in(0,1)$ such that $\|f(x)-f(y)\| \leq k\|x-y\|, x, y \in C$. A mapping $T: C \rightarrow C$ is called nonexpansive if $\|T x-T y\| \leq\|x-y\|, x, y \in C$.

In a real Hilbert space $H$, we have

$$
\|\lambda x+(1-\lambda) y\|^{2}=\lambda\|x\|^{2}+(1-\lambda)\|y\|^{2}-\lambda(1-\lambda)\|x-y\|^{2}
$$

for all $x, y \in H$ and $\lambda \in \mathbb{R}$. For every point $x \in H$, there exists the unique nearest point in $C$, denoted by $P_{C}(x)$, such that

$$
\left\|x-P_{C}(x)\right\| \leq\|x-y\|
$$

for all $y \in C$. $P_{C}$ is called the metric projection of $H$ onto $C$. It is well known that $P_{C}$ is nonexpansive and $P_{C}$ satisfies

$$
\left\langle x-y, P_{C}(x)-P_{C}(y)\right\rangle \geq\left\|P_{C}(x)-P_{C}(y)\right\|^{2}
$$

for every $x, y \in H$. Moreover, $P_{C}(x)$ is characterized by the properties:

$$
\|x-y\|^{2} \geq\left\|x-P_{C}(x)\right\|^{2}+\left\|y-P_{C}(x)\right\|^{2}
$$

and

$$
u=P_{C}(x) \Leftrightarrow\langle x-u, u-y\rangle \geq 0 \text { for all } x \in H, y \in C .
$$

In the context of the variational inequality problem for a nonlinear mapping $F$, this implies that

$$
u \in V I(C, F) \Leftrightarrow u=P_{C}(u-\lambda F u), \quad \text { for any } \lambda>0 .
$$

It is also well known that $H$ satisfies the Opial condition, that is, for any sequence $\left\{x_{n}\right\}$ with $x_{n} \rightarrow x$, the inequality

$$
\liminf _{n \rightarrow \infty}\left\|x_{n}-x\right\|<\liminf _{n \rightarrow \infty}\left\|x_{n}-y\right\|
$$

holds for every $y \in H$ with $y \neq x$.

A mapping $F$ of $C$ into $H$ is called $\alpha$-inverse-strongly monotone if there exists a constant $\alpha>0$ such that

$$
\langle x-y, F x-F y\rangle \geq \alpha\|F x-F y\|^{2}, \quad \forall x, y \in C .
$$

We know that if $F=I-T$, where $T$ is a nonexpansive mapping of $C$ into itself and $I$ is the identity mapping of $H$, then $F$ is $\frac{1}{2}$-inverse-strongly monotone and $V I(C, F)=$ $F(T)$. A mapping $F$ of $C$ into $H$ is called strongly monotone if there exists a positive real number $\eta$ such that

$$
\langle x-y, F x-F y\rangle \geq \eta\|x-y\|^{2}, \quad \forall x, y \in C .
$$

In such a case, we say $F$ is $\eta$-strongly monotone. If $F$ is $\eta$-strongly monotone and $\kappa$-Lipschitz continuous, that is, $\|F x-F y\| \leq \kappa|| x-y \|$ for all $x, y \in C$, then $F$ is $\frac{\eta}{\kappa^{2}}$-inverse-strongly monotone. If $F$ is an $\alpha$-inverse-strongly monotone mapping of $C$ into $H$, then it is obvious that $F$ is $\frac{1}{\alpha}$-Lipschitz continuous. We also have that for all $x$, $y \in C$ and $\lambda>0$, 


$$
\begin{aligned}
\|(I-\lambda F) x-(I-\lambda F) y\|^{2} & =\|(x-y)-\lambda(F x-F y)\|^{2} \\
& =\|x-y\|^{2}-2 \lambda\langle x-y, F x-F y\rangle+\lambda^{2}\|F x-F y\|^{2} \\
& \leq\|x-y\|^{2}+\lambda(\lambda-2 \alpha)\|F x-F y\|^{2} .
\end{aligned}
$$

Hence, if $\lambda \leq 2 \alpha$, then $I-\lambda F$ is a nonexpansive mapping of $C$ into $H$. The following result for the existence of solutions of the variational inequality problem for inversestrongly monotone mappings was given in Takahashi and Toyoda [25].

Proposition Let $C$ be a bounded closed convex subset of a real Hilbert space, and $F$ be an $\alpha$-inverse-strongly monotone mapping of $C$ into $H$. Then, $V I(C, F)$ is nonempty.

A set-valued mapping $Q: H \rightarrow 2^{H}$ is called monotone if for all $x, y \in H, f \in Q x$ and $g \in Q y$ imply $\langle x-y, f-g\rangle \geq 0$. A monotone mapping $Q: H \rightarrow 2^{H}$ is maximal if the graph $G(Q)$ of $Q$ is not properly contained in the graph of any other monotone mapping. It is known that a monotone mapping $Q$ is maximal if and only if for $(x, f) \in H$ $\times H,\langle x-y, f-g\rangle \geq 0$ for every $(y, g) \in G(Q)$ implies $f \in Q x$. Let $F$ be an inversestrongly monotone mapping of $C$ into $H$, and let $N_{C} v$ be the normal cone to $C$ at $v$, that is, $N_{C^{v}}=\{w \in H:\langle v-u, w\rangle \geq 0$, for all $u \in C\}$, and define

$$
Q v= \begin{cases}F v+N_{C} v, & v \in C \\ \emptyset & v \notin C .\end{cases}
$$

Then, $Q$ is maximal monotone and $0 \in Q v$ if and only if $v \in V I(C, F)$; see [26,27].

For solving the equilibrium problem for a bifunction $\Theta: C \times C \rightarrow \mathbb{R}$, let us assume that $\Theta$ and $\phi$ satisfy the following conditions:

(A1) $\Theta(x, x)=0$ for all $x \in C$;

(A2) $\Theta$ is monotone, that is, $\Theta(x, y)+\Theta(y, x) \leq 0$ for all $x, y \in C$;

(A3) for each $x, y, z \in C$,

$$
\lim _{t \downarrow 0} \Theta(t z+(1-t) x, y) \leq \Theta(x, y)
$$

(A4) for each $x \in C, y \alpha \Theta(x, y)$ is convex and lower semicontinuous;

(A5) For each $y \in C, x \alpha \Theta(x, y)$ is weakly upper semicontinuous;

(B1) For each $x \in H$ and $r>0$, there exists a bounded subset $D_{x} \subseteq C$ and $y_{x} \in C$ such that for any $z \in C \backslash D_{x}$,

$$
\Theta\left(z, y_{x}\right)+\varphi\left(y_{x}\right)-\varphi(z)+\frac{1}{r}\left\langle y_{x}-z, z-x\right\rangle<0 ;
$$

(B2) $C$ is a bounded set;

The following lemmas were given in [1,4].

Lemma 2.1 ([4]) Let $C$ be a nonempty closed convex subset of $H$, and $\Theta$ be a bifunction of $C \times C$ into $\mathbb{R}$ satisfying (A1)-(A4). Let $r>0$ and $x \in H$. Then, there exists $z \in C$ such that

$$
\Theta(z, y)+\frac{1}{r}\langle y-z, z-x\rangle \geq 0, \quad \forall y \in C .
$$

Lemma 2.2 ([1]) Let $C$ be a nonempty closed convex subset of $H$. Let $\Theta$ be a bifunction form $C \times C$ to $\mathbb{R}$ satisfying (A1)-(A5) and $\phi: C \rightarrow \mathbb{R}$ be a proper lower semicontinuous and convex function. For $r>0$ and $x \in H$, define a mapping $S_{r}: H \rightarrow C$ as follows: 


$$
S_{r}(x)=\left\{z \in C: \Theta(z, y)+\varphi(y)-\varphi(z)+\frac{1}{r}\langle y-z, z-x\rangle \geq 0, \quad \forall y \in C\right\}
$$

for all $z \in H$. Assume that either (B1) or (B2) holds. Then, the following hold:

(1) For each $x \in H, S_{r}(x) \neq \varnothing$;

(2) $S_{r}$ is single-valued;

(3) $S_{r}$ is firmly nonexpansive, that is, for any $x, y \in H$,

$$
\left\|S_{r} x-S_{r} y\right\|^{2} \leq\left\langle S_{r} x-S_{r} y, x-y\right\rangle ;
$$

(4) $F\left(S_{r}\right)=\operatorname{MEP}(\Theta, \phi)$;

(5) $\operatorname{MEP}(\Theta, \phi)$ is closed and convex.

We also need the following lemmas for the proof of our main results.

Lemma 2.3 ([23]) Let $\left\{s_{n}\right\}$ be a sequence of non-negative real numbers satisfying

$$
s_{n+1} \leq\left(1-\lambda_{n}\right) s_{n}+\beta_{n}, \quad n \geq 1,
$$

where $\left\{\lambda_{n}\right\}$ and $\left\{\beta_{n}\right\}$ satisfy the following conditions:

(i) $\left\{\lambda_{n}\right\} \subset[0,1]$ and $\sum_{n=1}^{\infty} \lambda_{n}=\infty$ or, equivalently, $\prod_{n=1}^{\infty}\left(1-\lambda_{n}\right)=0$,

(ii) $\lim \sup _{n \rightarrow \infty} \frac{\beta_{n}}{\lambda_{n}} \leq 0$ or $\sum_{n=1}^{\infty}\left|\beta_{n}\right|<\infty$,

Then, $\lim _{n \rightarrow \infty} s_{n}=0$.

Lemma 2.4 In a Hilbert space, there holds the inequality

$$
\|x+y\|^{2} \leq\|x\|^{2}+2\langle y, x+y\rangle, \quad \forall x, y \in H .
$$

Lemma 2.5 (Aoyama et al. [28]) Let $C$ be a nonempty closed convex subset of $H$ and $\left\{T_{n}\right\}$ be a sequence of nonexpansive mappings of $C$ into itself. Suppose that

$$
\sum_{n=1}^{\infty} \sup \left\{\left\|T_{n+1} z-T_{n} z\right\|: z \in C\right\}<\infty .
$$

Then, for each $y \in C,\left\{T_{n} y\right\}$ converges strongly to some point of $C$. Moreover, let $T$ be a mapping of $C$ into itself defined by $T y=\lim _{n \rightarrow \infty} T_{n} y$ for all $y \in C$. Then, $\lim _{n \rightarrow \infty}$ sup $\left\{|| T z-T_{n} z||: z \in C\right\}=0$.

The following lemma can be found in [3](see also Lemma 2.1 in [22]).

Lemma 2.6 Let $C$ be a nonempty closed convex subset of a real Hilbert space $H$ and $g: C \rightarrow \mathbb{R} \cup\{\infty\}$ be a proper lower semicontinuous differentiable convex function. If $x^{*}$ is a solution to the minimization problem

$$
g\left(x^{*}\right)=\inf _{x \in C} g(x),
$$

then

$$
\left\langle g^{\prime}(x), x-x^{*}\right\rangle \geq 0, \quad x \in C .
$$

In particular, if $x *$ solves the optimization problem

$$
\min _{x \in C} \frac{\mu}{2}\langle A x, x\rangle+\frac{1}{2}\|x-u\|^{2}-h(x),
$$


then

$$
\left\langle u+(\gamma f-(I+\mu A)) x^{*}, x-x^{*}\right\rangle \leq 0, \quad x \in C,
$$

where $h$ is a potential function for $\gamma f$.

\section{Main results}

In this section, we introduce a new composite iterative scheme for finding a common point of the set of solutions of the problem (1.1), the set of fixed points of a countable family of nonexpansive mappings, and the set of solutions of the problem (1.4) for an inverse-strongly monotone mapping.

Theorem 3.1 Let $C$ be a nonempty closed convex subset of a real Hilbert space $H$ such that $C \pm C \subset C$. Let $\Theta$ be a bifunction from $C \times C$ to $\mathbb{R}$ satisfying (A1)-(A5) and $\phi: C \rightarrow$ $\mathbb{R}$ be a lower semicontinuous and convex function. Let $F, B$ be two $\alpha$, $\beta$-inverse-strongly monotone mappings of $C$ into $H$, respectively. Let $\left\{T_{n}\right\}$ be a sequence of nonexpansive mappings of $C$ into itself such that $\Omega_{1}:=\bigcap_{n=1}^{\infty} F\left(T_{n}\right) \cap \operatorname{VI}(C, F) \cap \operatorname{GMEP}(\Theta, \varphi, B) \neq \emptyset$. Let $\mu>0$ and $\gamma>0$ be real numbers. Let $f$ be a contraction of $C$ into itself with constant $k$ $\in(0,1)$ and $A$ be a strongly positive bounded linear operator on $C$ with constant $\bar{\gamma} \in(0,1)$ such that $0<\gamma<\frac{(1+\mu) \bar{\gamma}}{k}$. Assume that either (B1) or (B2) holds. Let $u \in C$, and let $\left\{x_{n}\right\},\left\{y_{n}\right\}$, and $\left\{u_{n}\right\}$ be sequences generated by $x_{1} \in C$ and

$$
\left\{\begin{array}{l}
\Theta\left(u_{n}, y\right)+\left\langle B x_{n}, y-u_{n}\right\rangle+\varphi(y)-\varphi\left(u_{n}\right)+\frac{1}{r_{n}}\left\langle y-u_{n}, u_{n}-x_{n}\right\rangle \geq 0, \quad \forall y \in C, \\
y_{n}=\alpha_{n}\left(u+\gamma f\left(x_{n}\right)\right)+\left(I-\alpha_{n}(I+\mu A)\right) T_{n} P_{C}\left(u_{n}-\lambda_{n} F u_{n}\right), \\
x_{n+1}=\left(1-\beta_{n}\right) y_{n}+\beta_{n} T_{n} P_{C}\left(y_{n}-\lambda_{n} F y_{n}\right), \quad n \geq 1
\end{array}\right.
$$

where $\left\{\alpha_{n}\right\},\left\{\beta_{n}\right\} \subset[0,1], \lambda_{n} \in[a, b] \subset(0,2 \alpha)$ and $r_{n} \in[c, d] \subset(0,2 \beta)$. Let $\left\{\alpha_{n}\right\},\left\{\lambda_{n}\right\}$ and $\left\{\beta_{n}\right\}$ satisfy the following conditions:

(C1) $\alpha_{n} \rightarrow 0(n \rightarrow \infty) ; \sum_{n=1}^{\infty} \alpha_{n}=\infty$;

(C2) $\beta_{n} \subset[0, a)$ for all $n \geq 0$ and for some $a \in(0,1)$;

(C3) $\quad \sum_{n=1}^{\infty}\left|\alpha_{n+1}-\alpha_{n}\right|<\infty, \quad \sum_{n=1}^{\infty}\left|\beta_{n+1}-\beta_{n}\right|<\infty, \quad \sum_{n=1}^{\infty}\left|\lambda_{n+1}-\lambda_{n}\right|<\infty$, $\sum_{n=1}^{\infty}\left|r_{n+1}-r_{n}\right|<\infty$.

Suppose that $\sum_{n=1}^{\infty} \sup \left\{\left\|T_{n+1} z-T_{n} z\right\|: z \in D\right\}<\infty$ for any bounded subset $D$ of $C$. Let $T$ be a mapping of $C$ into itself defined by $T z=\lim _{n \rightarrow \infty} T_{n} z$ for all $z \in C$ and suppose that $F(T)=\bigcap_{n=1}^{\infty} F\left(T_{n}\right)$. Then $\left\{x_{n}\right\}$ and $\left\{u_{n}\right\}$ converge strongly to $q \in \Omega_{1}$, which is a solution of the optimization problem:

$$
\min _{x \in \Omega_{1}} \frac{\mu}{2}\langle A x, x\rangle+\frac{1}{2}\|x-u\|^{2}-h(x), \quad(\mathrm{OP} 1)
$$

where $h$ is a potential function for $\gamma f$.

Proof First, from $\alpha_{n} \rightarrow 0(n \rightarrow \infty)$ in the condition (C1), we assume, without loss of generality, that $\alpha_{n} \leq(1+\mu\|A\|)^{-1}$ and $2((1+\mu) \bar{\gamma}-\gamma k) \alpha_{n}<1$ for $n \geq 1$. We know that if $A$ is bounded linear self-adjoint operator on $H$, then

$$
\|A\|=\sup \{|\langle A u, u\rangle|: u \in H,\|u\|=1\} .
$$


Observe that

$$
\begin{aligned}
\left\langle\left(I-\alpha_{n}(I+\mu A)\right) u, u\right\rangle & =1-\alpha_{n}-\alpha_{n} \mu\langle A u, u\rangle \\
& \geq 1-\alpha_{n}-\alpha_{n} \mu\|A\| \\
& \geq 0,
\end{aligned}
$$

which is to say $I-\alpha_{n}(I+\mu A)$ is positive. It follows that

$$
\begin{aligned}
\left\|I-\alpha_{n}(I+\mu A)\right\| & =\sup \left\{\left\langle\left(I-\alpha_{n}(I+\mu A)\right) u, u\right\rangle: u \in H,\|u\|=1\right\} \\
& =\sup \left\{1-\alpha_{n}-\alpha_{n} \mu\langle A u, u\rangle: u \in H,\|u\|=1\right\} \\
& \leq 1-\alpha_{n}(1+\mu \bar{\gamma}) \\
& <1-\alpha_{n}(1+\mu) \bar{\gamma} .
\end{aligned}
$$

Let us divide the proof into several steps. From now on, we put $z_{n}=P_{C}\left(u_{n}-\lambda_{n} F u_{n}\right)$ and $w_{n}=P_{C}\left(y_{n}-\lambda_{n} F y_{n}\right)$.

Step 1: We show that $\left\{x_{n}\right\}$ is bounded. To this end, let $p \in \Omega_{1}:=\bigcap_{n=1}^{\infty} F\left(T_{n}\right) \cap V I(C, F) \cap \operatorname{GMEP}(\Theta, \varphi, B)$ and $\left\{S_{r_{n}}\right\}$ be a sequence of mappings defined as in Lemma 2.2. Then $p=T_{n} p=S_{r_{n}}\left(p-r_{n} B p\right)$ and $p=P_{C}\left(p-\lambda_{n} F p\right)$ from (2.2). From $z_{n}=P_{C}\left(u_{n}-\lambda_{n} F u_{n}\right)$ and the fact that $P_{C}$ and $I-\lambda_{n} F$ are nonexpansive, it follows that

$$
\left\|z_{n}-p\right\| \leq\left\|\left(I-\lambda_{n} F\right) u_{n}-\left(I-\lambda_{n} F\right) p\right\| \leq\left\|u_{n}-p\right\| .
$$

Also, by $u_{n}=S_{r_{n}}\left(x_{n}-r_{n} B x_{n}\right) \in C$ and the $\beta$-inverse-strongly monotonicity of $B$, we have with $r_{n} \in(0,2 \beta)$,

$$
\begin{aligned}
\left\|u_{n}-p\right\|^{2} & \leq\left\|x_{n}-r_{n} B x_{n}-\left(p-r_{n} B p\right)\right\|^{2} \\
& \leq\left\|x_{n}-p\right\|^{2}-2 r_{n}\left\langle x_{n}-p, B x_{n}-B p\right\rangle+r_{n}^{2}\left\|B x_{n}-B p\right\|^{2} \\
& \leq\left\|x_{n}-p\right\|^{2}+r_{n}\left(r_{n}-2 \beta\right) \beta\left\|B x_{n}-B p\right\|^{2} \\
& \leq\left\|x_{n}-p\right\|^{2},
\end{aligned}
$$

that is, $\left\|u_{n}-p\right\| \leq\left\|x_{n}-p\right\|$, and so

$$
\left\|z_{n}-p\right\| \leq\left\|x_{n}-p\right\| .
$$

Similarly, we have

$$
\left\|w_{n}-p\right\| \leq\left\|y_{n}-p\right\| .
$$

Now, set $\bar{A}=(I+\mu A)$. Then, from (IS) and (3.1), we obtain

$$
\begin{aligned}
& \left\|y_{n}-p\right\| \\
\leq & \left(1-(1+\mu \bar{\gamma}) \alpha_{n}\right)\left\|z_{n}-p\right\|+\alpha_{n}\|u\| \\
& +\alpha_{n} \gamma\left\|f\left(x_{n}\right)-f(p)\right\|+\alpha_{n}\|\gamma f(p)-\bar{A} p\| \\
\leq & \left(1-(1+\mu \bar{\gamma}) \alpha_{n}\right)\left\|z_{n}-p\right\|+\alpha_{n}\|u\|+\alpha_{n} \gamma k\left\|x_{n}-p\right\|+\alpha_{n}\|\gamma f(p)-\bar{A} p\| \\
\leq & \left(1-((1+\mu) \bar{\gamma}-\gamma k) \alpha_{n}\right)\left\|x_{n}-p\right\|+\alpha_{n}(\|\gamma f(p)-\bar{A} p\|+\|u\|) \\
= & \left(1-((1+\mu) \bar{\gamma}-\gamma k) \alpha_{n}\right)\left\|x_{n}-p\right\|+((1+\mu) \bar{\gamma}-\gamma k) \alpha_{n} \frac{\|\gamma f(p)-\bar{A} p\|+\|u\|}{(1+\mu) \bar{\gamma}-\gamma k} .
\end{aligned}
$$


From (3.2) and (3.3), it follows that

$$
\begin{aligned}
\left\|x_{n+1}-p\right\| & \leq\left(1-\beta_{n}\right)\left\|y_{n}-p\right\|+\beta_{n}\left\|w_{n}-p\right\| \\
& \leq\left(1-\beta_{n}\right)\left\|y_{n}-p\right\|+\beta_{n}\left\|y_{n}-p\right\| \\
& =\left\|y_{n}-p\right\| \\
& \leq \max \left\{\left\|x_{n}-p\right\|, \frac{\|\gamma f(p)-\bar{A} p\|+\|u\|}{(1+\mu) \bar{\gamma}-\gamma k}\right\} .
\end{aligned}
$$

By induction, it follows from (3.4) that

$$
\left\|x_{n}-p\right\| \leq \max \left\{\left\|x_{1}-p\right\|, \frac{\|\gamma f(p)-\bar{A} p\|+\|u\|}{(1+\mu) \bar{\gamma}-\gamma k}\right\}, \quad n \geq 1 .
$$

Therefore, $\left\{x_{n}\right\}$ is bounded. Hence $\left\{u_{n}\right\},\left\{y_{n}\right\},\left\{z_{n}\right\},\left\{w_{n}\right\},\left\{f\left(x_{n}\right)\right\},\left\{F u_{n}\right\},\left\{F y_{n}\right\}$, and $\left\{\bar{A} T_{n} z_{n}\right\}$ are bounded. Moreover, since $\left\|T_{n} z_{n}-p\right\| \leq\left\|x_{n}-p\right\|$ and $\left\|T_{n} w_{n}-p\right\| \leq \| y_{n}$ $p \|,\left\{T_{n} z_{n}\right\}$ and $\left\{T_{n} w_{n}\right\}$ are also bounded, and since $\alpha_{n} \rightarrow 0$ in the condition (C1), we have

$$
\left\|y_{n}-T_{n} z_{n}\right\|=\alpha_{n}\left\|\left(u+\gamma f\left(x_{n}\right)\right)-\bar{A} T_{n} z_{n}\right\| \rightarrow 0 \quad(\text { as } n \rightarrow \infty) .
$$

Step 2: We show that $\lim _{n \rightarrow \infty}|| x_{n+1}-x_{n} \|=0$. Indeed, since $I-\lambda_{n} F$ and $P_{C}$ are nonexpansive, we have

$$
\begin{aligned}
\left\|z_{n}-z_{n-1}\right\| & =\left\|P_{C}\left(u_{n}-\lambda_{n} F u_{n}\right)-P_{C}\left(u_{n-1}-\lambda_{n-1} F u_{n-1}\right)\right\| \\
& \leq\left\|\left(I-\lambda_{n} F\right) u_{n}-\left(I-\lambda_{n} F\right) u_{n-1}+\left(\lambda_{n}-\lambda_{n-1}\right) F u_{n-1}\right\| \\
& \leq\left\|u_{n}-u_{n-1}\right\|+\left|\lambda_{n}-\lambda_{n-1}\right|\left\|F u_{n-1}\right\| .
\end{aligned}
$$

Similarly, we get

$$
\left\|w_{n}-w_{n-1}\right\| \leq|| y_{n}-y_{n-1}\left\|+\left|\lambda_{n}-\lambda_{n-1}\right|\right\| F y_{n-1} \| .
$$

On the other hand, from $u_{n-1}=S_{r_{n-1}}\left(x_{n-1}-r_{n} B x_{n-1}\right)$ and $u_{n}=S_{r_{n}}\left(x_{n}-r_{n} B x_{n}\right)$, it follows that

$$
\begin{aligned}
\Theta\left(u_{n-1}, y\right) & +\left\langle B x_{n-1}, y-u_{n-1}\right\rangle \\
& +\varphi(y)-\varphi\left(u_{n-1}\right)+\frac{1}{r_{n-1}}\left\langle y-u_{n-1}, u_{n-1}-x_{n-1}\right\rangle \geq 0, \quad \forall y \in C,
\end{aligned}
$$

and

$$
\begin{aligned}
\Theta\left(u_{n}, y\right) & +\left\langle B x_{n}, y-u_{n}\right\rangle \\
& +\varphi(y)-\varphi\left(u_{n}\right)+\frac{1}{r_{n}}\left\langle y-u_{n}, u_{n}-x_{n}\right\rangle \geq 0, \quad \forall y \in C .
\end{aligned}
$$

Substituting $y=u_{n}$ into (3.8) and $y=u_{n-1}$ into (3.9), we obtain

$$
\Theta\left(u_{n-1}, u_{n}\right)+\left\langle B x_{n-1}, u_{n}-u_{n-1}\right\rangle+\varphi\left(u_{n}\right)-\varphi\left(u_{n-1}\right)+\frac{1}{r_{n-1}}\left\langle u_{n}-u_{n-1}, u_{n-1}-x_{n-1}\right\rangle \geq 0
$$

and

$$
\Theta\left(u_{n}, u_{n-1}\right)+\left\langle B x_{n}, u_{n-1}-u_{n}\right\rangle+\varphi\left(u_{n-1}\right)-\varphi\left(u_{n}\right)+\frac{1}{r_{n}}\left\langle u_{n-1}-u_{n}, u_{n}-x_{n}\right\rangle \geq 0 .
$$


From (A2), we have

$$
\left\langle u_{n}-u_{n-1}, B x_{n-1}-B x_{n}+\frac{u_{n-1}-x_{n-1}}{r_{n-1}}-\frac{u_{n}-x_{n}}{r_{n}}\right\rangle \geq 0,
$$

and then

$$
\left\langle u_{n}-u_{n-1}, r_{n-1}\left(B x_{n-1}-B x_{n}\right)+u_{n-1}-x_{n-1}-\frac{r_{n-1}}{r_{n}}\left(u_{n}-x_{n}\right)\right\rangle \geq 0 .
$$

Hence, it follows that

$$
\begin{aligned}
\left\langle u_{n}-u_{n-1},\left(I-r_{n-l} B\right) x_{n}-\left(I-r_{n-1} B\right) x_{n-1}\right. \\
\left.+u_{n-1}-u_{n}+u_{n}-x_{n}-\frac{r_{n-1}}{r_{n}}\left(u_{n}-x_{n}\right)\right\rangle \geq 0 .
\end{aligned}
$$

Without loss of generality, let us assume that there exists a real number $c$ such that $r_{n}>c>0$ for all $n \geq 1$. Then, by (3.10) and the fact that $\left(I-r_{n-1} B\right)$ is nonexpansive, we have

$$
\begin{aligned}
& \left\|u_{n}-u_{n-1}\right\|^{2} \\
\leq & \left\langle u_{n}-u_{n-1},\left(I-r_{n-1} B\right) x_{n}-\left(I-r_{n-1} B\right) x_{n-1}+\left(1-\frac{r_{n-1}}{r_{n}}\right)\left(u_{n}-x_{n}\right)\right\rangle \\
\leq & \left\|u_{n}-u_{n-1}\right\|\left\{\left\|\left(I-r_{n-1} B\right) x_{n}-\left(I-r_{n-1} B\right) x_{n-1}\right\|+\left|1-\frac{r_{n-1}}{r_{n}}\right|\left\|u_{n}-x_{n}\right\|\right\} \\
\leq & \left\|u_{n}-u_{n-1}\right\|\left\{\left\|x_{n}-x_{n-1}\right\|+\left|1-\frac{r_{n-1}}{r_{n}}\right|\left\|u_{n}-x_{n}\right\|\right\},
\end{aligned}
$$

which implies that

$$
\begin{aligned}
\left\|u_{n}-u_{n-1}\right\| & \leq\left\|x_{n}-x_{n-1}\right\|+\frac{1}{r_{n}}\left|r_{n}-r_{n-1}\right||| u_{n}-x_{n}|| \\
& \leq\left\|x_{n}-x_{n-1}\right\|+\frac{M_{1}}{c}\left|r_{n}-r_{n-1}\right|,
\end{aligned}
$$

where $M_{1}=\sup \left\{\left\|u_{n}-x_{n}\right\|: n \geq 1\right\}$. Substituting (3.11) into (3.6), we have

$$
|| z_{n}-z_{n-1}|| \leq|| x_{n}-x_{n-1}||+\frac{M_{1}}{c}\left|r_{n}-r_{n-1}\right|+\left|\lambda_{n}-\lambda_{n-1}\right| \| F u_{n-1}|| .
$$

Simple calculations show that

$$
\begin{aligned}
y_{n}-y_{n-1}= & \left(\alpha_{n}-\alpha_{n-1}\right)\left(u+\gamma f\left(x_{n-1}\right)-\bar{A} T_{n-1} z_{n-1}\right)+\alpha_{n} \gamma\left(f\left(x_{n}\right)-f\left(x_{n-1}\right)\right) \\
& +\left(I-\alpha_{n} \bar{A}\right)\left(T_{n} z_{n}-T_{n} z_{n-1}\right)+\left(I-\alpha_{n} \bar{A}\right)\left(T_{n} z_{n-1}-T_{n-1} z_{n-1}\right) .
\end{aligned}
$$


Hence, by (3.11) and (3.12), we obtain

$$
\begin{aligned}
\left\|y_{n}-y_{n-1}\right\| \leq & \left|\alpha_{n}-\alpha_{n-1}\right|\left(\|u\|+\gamma\left\|f\left(x_{n-1}\right)\right\|+\|\bar{A}\| \mid\left\|T_{n-1} z_{n-1}\right\|\right) \\
& +\alpha_{n} \gamma k\left\|x_{n}-x_{n-1}\right\|+\left(1-(1+\mu) \bar{\gamma} \alpha_{n}\right)\left\|z_{n}-z_{n-1}\right\| \\
& +\left(1-(1+\mu) \bar{\gamma} \alpha_{n}\right)\left\|T_{n} z_{n-1}-T_{n-1} z_{n-1}\right\| \\
\leq & \left|\alpha_{n}-\alpha_{n-1}\right|\left(\|u\|+\gamma\left\|f\left(x_{n-1}\right)\right\|+\|\bar{A}\|\left\|T_{n-1} z_{n-1}\right\|\right) \\
& +\alpha_{n} \gamma k\left\|x_{n}-x_{n-1}\right\|+\left(1-(1+\mu) \bar{\gamma} \alpha_{n}\right)|| x_{n}-x_{n-1} \| \\
& +\frac{M_{1}}{c}\left|r_{n}-r_{n-1}\right|+\left|\lambda_{n}-\lambda_{n-1}\right|\left\|F u_{n-1}\right\|+\sup _{z \in D_{1}}\left\|T_{n} z-T_{n-1} z\right\|
\end{aligned}
$$

where $D_{1}$ is a bounded subset of $C$ containing $\left\{z_{n}\right\}$. Also observe that

$$
\begin{aligned}
x_{n+1}-x_{n}= & \left(1-\beta_{n}\right)\left(y_{n}-y_{n-1}\right)+\left(\beta_{n}-\beta_{n-1}\right)\left(T_{n-1} w_{n-1}-y_{n-1}\right) \\
& +\beta_{n}\left(T_{n} w_{n}-T_{n} w_{n-1}\right)+\beta_{n}\left(T_{n} w_{n-1}-T_{n-1} w_{n-1}\right) .
\end{aligned}
$$

By (3.7), (3.13) and (3.14), we have

$$
\begin{aligned}
& \left\|x_{n+1}-x_{n}\right\| \\
& \leq\left(1-\beta_{n}\right)|| y_{n}-y_{n-1}||+\left|\beta_{n}-\beta_{n-1}\right|\left(|| T_{n-1} x_{n-1}||+|| y_{n-1}||\right) \\
& +\beta_{n}|| w_{n}-w_{n-1}\left\|+\beta_{n}\right\| T_{n} w_{n-1}-T_{n-1} w_{n-1} \| \\
& \leq\left(1-\beta_{n}\right)|| y_{n}-y_{n-1}||+\beta_{n}|| y_{n}-y_{n-1}||+\beta_{n}\left|\lambda_{n}-\lambda_{n-1}\right||| F y_{n-1} \| \\
& +\left|\beta_{n}-\beta_{n-1}\right|\left(|| T_{n-1} w_{n-1}||+|| y_{n-1}||\right)+\beta_{n}|| T_{n} w_{n-1}-T_{n-1} w_{n-1} \| \\
& \leq|| y_{n}-y_{n-1}||+\left|\lambda_{n}-\lambda_{n-1}\right||| F \gamma_{n-1} \| \\
& +\left|\beta_{n}-\beta_{n-1}\right|\left(|| T_{n-1} w_{n-1}||+|| \gamma_{n-1}||\right)+|| T_{n} w_{n-1}-T_{n-1} w_{n-1}|| \\
& \leq\left(1-((1+\mu) \bar{\gamma}-\gamma k) \alpha_{n}\right)\left\|x_{n}-x_{n-1}\right\| \\
& +\mid\left(\alpha_{n}-\alpha_{n-1} \mid\left(|| u||+\gamma|| f\left(x_{n-1}\right)||+|| \bar{A} \||| T_{n-1} z_{n-1}||\right)\right. \\
& +\left|\lambda_{n}-\lambda_{n-1}\right|\left(|| F y_{n-1}||+|| F u_{n-1}||\right)+\frac{M_{1}}{c}\left|r_{n}-r_{n-1}\right| \\
& +\left|\beta_{n}-\beta_{n-1}\right|\left(|| T_{n-1} w_{n-1}||+|| \gamma_{n-1}||\right)+2 \sup _{z \in D_{2}}\left\{|| T_{n} z-T_{n-1} z||\right. \\
& \leq\left(1-((1+\mu) \bar{\gamma}-\gamma k) \alpha_{n}\right)|| x_{n}-x_{n-1}||+\frac{M_{1}}{c}\left|r_{n}-r_{n-1}\right| \\
& +M_{2}\left|\alpha_{n}-\alpha_{n-1}\right|+M_{3}\left|\lambda_{n}-\lambda_{n-1}\right|+M_{4}\left|\beta_{n}-\beta_{n-1}\right| \\
& +2 \sup _{z \in D_{2}}\left\|T_{n} z-T_{n-1} z\right\| \text {, }
\end{aligned}
$$

where $D_{2}$ is a bounded subset of $C$ containing $\left\{z_{n}\right\}$ and $\left\{w_{n}\right\}$, $M_{2}=\sup \left\{\|u\|+\gamma\left\|f\left(x_{n}\right)\right\|+\|\bar{A}\|\left\|T_{n} z_{n}\right\|: n \geq 1\right\}, M_{3}=\sup \left\{\left\|F y_{n}\right\|+\left\|F u_{n}\right\|: n \geq 1\right\}$, and $M_{4}=\sup \left\{\left\|T_{n} w_{n}\right\|+\left\|y_{n}\right\|: n \geq 1\right\}$. From the conditions (C1) and (C3) and the condition $\sum_{n=1}^{\infty} \sup \left\{\left\|T_{n+1} z-T_{z}\right\|: z \in D\right\}<\infty$ for any bounded subset $D$ of $C$, it is easy to see that

$$
\lim _{n \rightarrow \infty}((1+\mu) \bar{\gamma}-\gamma k) \alpha_{n}=0, \quad \sum_{n=1}^{\infty}((1+\mu) \bar{\gamma}-\gamma k) \alpha_{n}=\infty,
$$


and

$$
\begin{aligned}
& \sum_{n=2}^{\infty}\left(\frac{M_{1}}{c}\left|r_{n}-r_{n-1}\right|+M_{2}\left|\alpha_{n}-\alpha_{n-1}\right|+M_{3}\left|\lambda_{n}-\lambda_{n-1}\right|\right. \\
& \left.+M_{4}\left|\beta_{n}-\beta_{n-1}\right|+2 \sup _{z \in D_{2}}|| T_{n} z-T_{n-1} z||\right)<\infty .
\end{aligned}
$$

Applying Lemma 2.3 to (3.15), we obtain

$$
\lim _{n \rightarrow \infty}\left\|x_{n+1}-x_{n}\right\|=0 .
$$

Moreover, from (3.11), it follows that

$$
\lim _{n \rightarrow \infty}\left\|u_{n+1}-u_{n}\right\|=0
$$

From (3.12) and (3.13), we also have

$$
\lim _{n \rightarrow \infty}\left\|z_{n+1}-z_{n}\right\|=0 \text { and } \lim _{n \rightarrow \infty}\left\|y_{n+1}-y_{n}\right\|=0
$$

Step 3: We show that $\lim _{n-\infty}\left\|x_{n}-u_{n}\right\|=0$. To this end, let $p \in \Omega_{1}$. Since $S_{r_{n}}$ is firmly nonexpansive and $u_{n}=S_{r_{n}}\left(x_{n}-r_{n} B x_{n}\right)$, we have

$$
\begin{aligned}
\left\|u_{n}-p\right\|^{2} \leq & \left\langle S_{r_{n}}\left(x_{n}-r_{n} B x_{n}\right)-S_{r_{n}}\left(p-r_{n} B p\right), x_{n}-r_{n} B x_{n}-\left(p-r_{n} B p\right)\right\rangle \\
= & \frac{1}{2}\left\{\left\|u_{n}-p\right\|^{2}+\left\|x_{n}-r_{n} B x_{n}-\left(p-r_{n} B p\right)\right\|^{2}\right\} \\
& \quad-\frac{1}{2}\left\{\left\|x_{n}-r_{n} B x_{n}-\left(p-r_{n} B p\right)-\left(u_{n}-p\right)\right\|^{2}\right\} \\
& \leq \frac{1}{2}\left\{\left\|u_{n}-p\right\|^{2}+\left\|x_{n}-p\right\|^{2}-\left\|x_{n}-u_{n}-r_{n}\left(B x_{n}-B p\right)\right\|^{2}\right\} \\
\leq & \frac{1}{2}\left\{\left\|u_{n}-p\right\|^{2}+\left\|x_{n}-p\right\|^{2}-\left\|x_{n}-u_{n}\right\|^{2}\right. \\
& \left.\quad+2 r_{n}\left\langle B x_{n}-B p, x_{n}-u_{n}\right\rangle-r_{n}^{2}\left\|B x_{n}-B p\right\|^{2}\right\} .
\end{aligned}
$$

Hence,

$$
\begin{aligned}
& \left\|u_{n}-p\right\|^{2} \\
\leq & \left\|x_{n}-p\right\|^{2}-\left\|x_{n}-u_{n}\right\|^{2}+2 r_{n}\left\langle B x_{n}-B p, x_{n}-u_{n}\right\rangle-r_{n}^{2}\left\|B x_{n}-B p\right\|^{2} \\
\leq & \left\|x_{n}-p\right\|^{2}-\left\|x_{n}-u_{n}\right\|^{2}+2 r_{n}\left\langle B x_{n}-B p, x_{n}-u_{n}\right\rangle \\
\leq & \left\|x_{n}-p\right\|^{2}-\left\|x_{n}-u_{n}\right\|^{2}+2 r_{n}\left\|B x_{n}-B p\right\|\left\|x_{n}-u_{n}\right\| .
\end{aligned}
$$

On the other hand, since $z_{n}=P_{C}\left(u_{n}-\lambda_{n} F u_{n}\right)$, we get

$$
\begin{aligned}
\left\|z_{n}-p\right\|^{2} & \leq\left\|\left(I-\lambda_{n} F\right) u_{n}-\left(I-\lambda_{n} F\right) p\right\|^{2} \\
& \leq\left\|u_{n}-p\right\|^{2} .
\end{aligned}
$$


From (3.16), (3.17), and the convexity of \|\|$^{2}$, we obtain

$$
\begin{aligned}
\left\|y_{n}-p\right\|^{2} \leq & \alpha_{n}\left\|u+\gamma f\left(x_{n}\right)+(I-\bar{A}) T_{n} z_{n}-p\right\|^{2}+\left(1-\alpha_{n}\right)\left\|T_{n} z_{n}-p\right\|^{2} \\
\leq & \alpha_{n}\left\|u+\gamma f\left(x_{n}\right)+(I-\bar{A}) T_{n} z_{n}-p\right\|^{2}+\left(1-\alpha_{n}\right)\left\|z_{n}-p\right\|^{2} \\
\leq & \alpha_{n}\left\|u+\gamma f\left(x_{n}\right)+(I-\bar{A}) T_{n} z_{n}-p\right\|^{2}+\left(1-\alpha_{n}\right)\left\|u_{n}-p\right\|^{2} \\
\leq & \alpha_{n}\left\|u+\gamma f\left(x_{n}\right)+(I-\bar{A}) T_{n} z_{n}-p\right\|^{2} \\
& +\left(1-\alpha_{n}\right)\left\{\left\|x_{n}-p\right\|^{2}-\left\|x_{n}-u_{n}\right\|^{2}+2 r_{n}\left\|x_{n}-u_{n}\right\|\left\|B x_{n}-B p\right\|\right\} \\
\leq & \alpha_{n}\left\|u+\gamma f\left(x_{n}\right)+(I-\bar{A}) T_{n} z_{n}-p\right\|^{2}+\left\|x_{n}-p\right\|^{2} \\
& -\left(1-\alpha_{n}\right)\left\|x_{n}-u_{n}\right\|^{2}+2\left(1-\alpha_{n}\right) r_{n}\left\|x_{n}-u_{n}\right\|\left\|B x_{n}-B p\right\| .
\end{aligned}
$$

On the another hand, we note that

$$
\begin{aligned}
\left\|u_{n}-p\right\|^{2} & \leq\left\|\left(x_{n}-r_{n} B x_{n}\right)-\left(p-r_{n} B p\right)\right\|^{2} \\
& \leq\left\|x_{n}-p\right\|^{2}-2 r_{n}\left\langle x_{n}-p, B x_{n}-B p\right\rangle+r_{n}^{2}\left\|B x_{n}-B p\right\|^{2} \\
& \leq\left\|x_{n}-p\right\|^{2}-2 r_{n} \beta\left\|B x_{n}-B p\right\|^{2}+r_{n}^{2}\left\|B x_{n}-B p\right\|^{2} .
\end{aligned}
$$

Using the convexity of \|\|$^{2}$, (3.2), (3.18), and (3.19), we have

$$
\begin{aligned}
\left\|x_{n+1}-p\right\|^{2} \leq & \left\|y_{n}-p\right\|^{2} \\
\leq & \alpha_{n}\left\|u+\gamma f\left(x_{n}\right)+(I-\bar{A}) T_{n} z_{n}-p\right\|^{2}+\left(1-\alpha_{n}\right)\left\|u_{n}-p\right\|^{2} \\
\leq & \alpha_{n}\left\|u+\gamma f\left(x_{n}\right)+(I-\bar{A}) T_{n} z_{n}-p\right\|^{2} \\
& +\left(1-\alpha_{n}\right)\left\{\left\|x_{n}-p\right\|^{2}-2 r_{n} \beta\left\|B x_{n}-B p\right\|^{2}+r_{n}^{2}\left\|B x_{n}-B p\right\|^{2}\right\} \\
\leq & \alpha_{n}\left\|u+\gamma f\left(x_{n}\right)+(I-\bar{A}) T_{n} z_{n}-p\right\|^{2}+\left\|x_{n}-p\right\|^{2} \\
& +\left(1-\alpha_{n}\right) r_{n}\left(r_{n}-2 \beta\right)\left\|B x_{n}-B p\right\|^{2} .
\end{aligned}
$$

Hence, we have

$$
\begin{aligned}
& \left(1-\alpha_{n}\right) c(2 \beta-d)\left\|B x_{n}-B p\right\|^{2} \\
\leq & \left(1-\alpha_{n}\right) r_{n}\left(2 \beta-r_{n}\right)\left\|B x_{n}-B p\right\|^{2} \\
\leq & \alpha_{n}\left\|u+\gamma f\left(x_{n}\right)+(I-\bar{A}) T_{n} z_{n}-p\right\|^{2}+\left(\left\|x_{n}-p\right\|^{2}-\left\|x_{n+1}-p\right\|^{2}\right) \\
\leq & \alpha_{n}\left\|u+\gamma f\left(x_{n}\right)+(I-\bar{A}) T_{n} z_{n}-p\right\|^{2}+\left\|x_{n}-x_{n+1}\right\|\left(\left\|x_{n}-p\right\|+\left\|x_{n+1}-p\right\|\right) .
\end{aligned}
$$

From the condition $(\mathrm{C} 1),\left\{r_{n}\right\} \subset[c, d] \subset(0,2 \beta)$ and Step 2, it follows that

$$
\lim _{n \rightarrow \infty}\left\|B x_{n}-B p\right\|^{2}=0 .
$$

Also, by (3.16) and (3.20), we have

$$
\begin{aligned}
\left\|x_{n+1}-p\right\|^{2} \leq & \left\|y_{n}-p\right\|^{2} \\
\leq & \alpha_{n}\left\|u+\gamma f\left(x_{n}\right)+(I-\bar{A}) T_{n} z_{n}-p\right\|^{2}+\left(1-\alpha_{n}\right)\left\|u_{n}-p\right\|^{2} \\
\leq & \alpha_{n}\left\|u+\gamma f\left(x_{n}\right)+(I-\bar{A}) T_{n} z_{n}-p\right\|^{2} \\
& +\left(1-\alpha_{n}\right)\left\{\left\|x_{n}-p\right\|^{2}-\left\|x_{n}-u_{n}\right\|^{2}+2 r_{n}\left\|x_{n}-u_{n}\right\|\left\|B x_{n}-B p\right\|\right\} \\
\leq & \alpha_{n}\left\|u+\gamma f\left(x_{n}\right)+(I-\bar{A}) T_{n} z_{n}-p\right\|^{2}+\left\|x_{n}-p\right\|^{2} \\
& -\left(1-\alpha_{n}\right)\left\|x_{n}-u_{n}\right\|^{2}+2 r_{n}\left(1-\alpha_{n}\right)\left\|x_{n}-u_{n}\right\|\left\|B x_{n}-B p\right\|,
\end{aligned}
$$


and so

$$
\begin{aligned}
\left(1-\alpha_{n}\right)\left\|x_{n}-u_{n}\right\|^{2} \leq & \alpha_{n}\left\|u+\gamma f\left(x_{n}\right)+(I-\bar{A}) T_{n} z_{n}-p\right\|^{2}+\left\|x_{n}-p\right\|^{2} \\
& -\left\|x_{n+1}-p\right\|^{2}+2 r_{n}\left(1-\alpha_{n}\right)\left\|x_{n}-u_{n}\right\|\left\|B x_{n}-B p\right\| \\
\leq & \alpha_{n} M_{5}+\left\|x_{n}-x_{n+1}\right\|\left(\left\|x_{n}-p\right\|+\left\|x_{n+1}-p\right\|\right) \\
& +2 r_{n}\left(1-\alpha_{n}\right)\left\|x_{n}-u_{n}\right\|\left\|B x_{n}-B p\right\|
\end{aligned}
$$

where $M_{5}=\sup \left\{\left\|u+\gamma f\left(x_{n}\right)+(I-\bar{A}) T_{n} z_{n}-p\right\|^{2}: n \geq 1\right\}$. Since $\left\|x_{n+1}-x_{n}\right\| \rightarrow 0, \alpha_{n}$ $\rightarrow 0$, and $\left\|B x_{n}-B p\right\| \rightarrow 0$ as $n \rightarrow \infty$, we obtain

$$
\lim _{n \rightarrow \infty}\left\|x_{n}-u_{n}\right\|=0
$$

Moreover, since $\lim \inf _{n \rightarrow \infty} r_{n}>0$, we also have

$$
\lim _{n \rightarrow \infty} \frac{\left\|x_{n}-u_{n}\right\|}{r_{n}}=\lim _{n \rightarrow \infty} \frac{1}{r_{n}}\left\|x_{n}-u_{n}\right\|=0 .
$$

Step 4: We show that $\lim _{n \rightarrow \infty}|| x_{n}-T_{n} z_{n} \|=0$ and $\lim _{n-\infty}|| x_{n}-y_{n}||=0$. Indeed, since $z_{n}=P_{C}\left(u_{n}-\lambda_{n} F u_{n}\right)$ and $w_{n}=P_{C}\left(y_{n}-\lambda_{n} F y_{n}\right)$, we obtain with the condition (C2)

$$
\begin{aligned}
\left\|x_{n+1}-y_{n}\right\| & \leq \beta_{n}\left(\left\|T_{n} z_{n}-T_{n} w_{n}\right\|+\left\|y_{n}-T_{n} z_{n}\right\|\right) \\
& \leq a\left(\left\|z_{n}-w_{n}\right\|+\left\|y_{n}-T_{n} z_{n}\right\|\right) \\
& \leq a\left(\left\|u_{n}-y_{n}\right\|+\left\|y_{n}-T_{n} z_{n}\right\|\right) \\
& \leq a\left(\left\|u_{n}-x_{n}\right\|+\left\|x_{n}-x_{n+1}\right\|+\left\|x_{n+1}-y_{n}\right\|+\left\|y_{n}-T_{n} z_{n}\right\|\right),
\end{aligned}
$$

which implies that

$$
\left\|x_{n+1}-y_{n}\right\| \leq \frac{a}{1-a}\left(\left\|u_{n}-x_{n}\right\|+\left\|x_{n}-x_{n+1}\right\|+\left\|y_{n}-T_{n} z_{n}\right\|\right) .
$$

Thus, from (3.5), Step 2, and Step 3, we have

$$
\left\|x_{n+1}-y_{n}\right\| \rightarrow 0 \text { as } n \rightarrow \infty \text {. }
$$

Also we have

$$
\left\|x_{n}-y_{n}\right\| \leq\left\|x_{n}-x_{n+1}\right\|+\left\|x_{n+1}-y_{n}\right\| \rightarrow 0 \text { as } n \rightarrow \infty
$$

Since $\lim _{n \rightarrow \infty}\left\|y_{n}-T_{n} z_{n}\right\|=0$ by (3.5) in Step 1, we obtain

$$
\left\|x_{n}-T_{n} z_{n}\right\| \leq\left\|x_{n}-y_{n}\right\|+\left\|y_{n}-T_{n} z_{n}\right\| \rightarrow 0 \text { as } n \rightarrow \infty .
$$

Step 5: We show that $\lim _{n \rightarrow \infty}\left\|T_{n} z_{n}-z_{n}\right\|=0$. Let $p \in \Omega_{1}$. Using the convexity of $\|$ $\|^{2}$, we compute 


$$
\begin{aligned}
\left\|y_{n}-p\right\|^{2} \leq & \alpha_{n}\left\|u+\gamma f\left(x_{n}\right)+(I-\bar{A}) T_{n} z_{n}-p\right\|^{2}+\left(1-\alpha_{n}\right)\left\|T_{n} z_{n}-p\right\|^{2} \\
\leq & \alpha_{n}\left\|u+\gamma f\left(x_{n}\right)+(I-\bar{A}) T_{n} z_{n}-p\right\|^{2}+\left(1-\alpha_{n}\right)\left\|z_{n}-p\right\|^{2} \\
= & \alpha_{n}\left\|u+\gamma f\left(x_{n}\right)+(I-\bar{A}) T_{n} z_{n}-p\right\|^{2} \\
& +\left(1-\alpha_{n}\right)\left\|P_{C}\left(u_{n}-\lambda_{n} F u_{n}\right)-P_{C}\left(p-\lambda_{n} F p\right)\right\|^{2} \\
\leq & \alpha_{n}\left\|u+\gamma f\left(x_{n}\right)+(I-\bar{A}) T_{n} z_{n}-p\right\|^{2} \\
& +\left(1-\alpha_{n}\right)\left\|\left(u_{n}-p\right)-\lambda_{n}\left(F u_{n}-F p\right)\right\|^{2} \\
= & \alpha_{n}\left\|u+\gamma f\left(x_{n}\right)+(I-\bar{A}) T_{n} z_{n}-p\right\| \|^{2} \\
& +\left(1-\alpha_{n}\right)\left\{\left\|u_{n}-p\right\|^{2}-2 \lambda_{n}\left\langle u_{n}-p, F u_{n}-F p\right\rangle+\lambda_{n}^{2}\left\|F u_{n}-F p\right\|^{2}\right\} \\
\leq & \alpha_{n}\left\|u+\gamma f\left(x_{n}\right)+(I-\bar{A}) T_{n} z_{n}-p\right\|^{2} \\
& +\left(1-\alpha_{n}\right)\left\{\left\|u_{n}-p\right\|^{2}-2 \lambda_{n} \alpha\left\|F u_{n}-F p\right\|^{2}+\lambda_{n}^{2}\left\|F u_{n}-F p\right\|^{2}\right\} \\
\leq & \alpha_{n}\left\|u+\gamma f\left(x_{n}\right)+(I-\bar{A}) T_{n} z_{n}-p\right\|^{2} \\
& +\left\|x_{n}-p\right\|^{2}+\left(1-\alpha_{n}\right) \lambda_{n}\left(\lambda_{n}-2 \alpha\right)\left\|F u_{n}-F p\right\|^{2} .
\end{aligned}
$$

Using (3.24), we obtain

$$
\begin{aligned}
\left\|x_{n+1}-p\right\|^{2} \leq & \left\|y_{n}-p\right\|^{2} \\
\leq & \alpha_{n}\left\|u+\gamma f\left(x_{n}\right)+(I-\bar{A}) T_{n} z_{n}-p\right\|^{2} \\
& +\left\|x_{n}-p\right\|^{2}+\left(1-\alpha_{n}\right) \lambda_{n}\left(\lambda_{n}-2 \alpha\right)\left\|F u_{n}-F p\right\|^{2} .
\end{aligned}
$$

Hence, we have

$$
\begin{aligned}
& \left(1-\alpha_{n}\right) a(2 \alpha-b)\left\|F u_{n}-F p\right\|^{2} \\
\leq & \left(1-\alpha_{n}\right) \lambda_{n}\left(2 \alpha-\lambda_{n}\right)\left\|F u_{n}-F p\right\|^{2} \\
\leq & \alpha_{n}\left\|u+\gamma f\left(x_{n}\right)+(I-\bar{A}) T_{n} z_{n}-p\right\|^{2} \\
& +\left(\left\|x_{n}-p\right\|-\left\|x_{n+1}-p\right\|\right)\left(\left\|x_{n}-p\right\|+\left\|x_{n+1}-p\right\|\right) \\
\leq & \alpha_{n} M_{5}+\left\|x_{n}-x_{n+1}\right\|\left(\left\|x_{n}-p\right\|+\left\|x_{n+1}-p\right\|\right) .
\end{aligned}
$$

where $M_{5}=\sup \left\{\left\|u+\gamma f\left(x_{n}\right)+(I-\bar{A}) T_{n} z_{n}-p\right\|^{2}: n \geq 1\right\}$. From the condition (C1), $\lambda_{n} \in[a, b] \subset(0,2 \alpha)$, and Step 2, it follows that

$$
\lim _{n \rightarrow \infty}\left\|F u_{n}-F p\right\|=0 .
$$

On the other hand, using $z_{n}=P_{C}\left(u_{n}-\lambda_{n} F u_{n}\right)$ and (2.1), we observe that

$$
\begin{aligned}
\left\|z_{n}-p\right\|^{2} \leq & \left\langle\left(u_{n}-\lambda_{n} F u_{n}\right)-\left(p-\lambda_{n} F p\right), z_{n}-p\right\rangle \\
\leq & \frac{1}{2}\left\{\left\|u_{n}-p\right\|^{2}+\left\|z_{n}-p\right\|^{2}-\left\|\left(u_{n}-z_{n}\right)-\lambda_{n}\left(F u_{n}-F p\right)\right\|^{2}\right\} \\
\leq & \frac{1}{2}\left\{\left\|x_{n}-p\right\|^{2}+\left\|z_{n}-p\right\|^{2}-\left\|u_{n}-z_{n}\right\|^{2}\right. \\
& \left.+2 \lambda_{n}\left\langle u_{n}-z_{n}, F u_{n}-F p\right\rangle-\lambda_{n}^{2}\left\|F u_{n}-F p\right\|^{2}\right\},
\end{aligned}
$$

and so

$$
\begin{aligned}
\left\|z_{n}-p\right\|^{2} \leq & \left\|x_{n}-p\right\|^{2}-\left\|u_{n}-z_{n}\right\|^{2} \\
& +2 \lambda_{n}\left\langle u_{n}-z_{n}, F u_{n}-F p\right\rangle-\lambda_{n}^{2}\left\|F u_{n}-F p\right\|^{2}
\end{aligned}
$$


Thus, from (3.26), we have

$$
\begin{aligned}
\left\|y_{n}-p\right\|^{2} \leq & \alpha_{n}\left\|u+\gamma f\left(x_{n}\right)+(I-\bar{A}) T_{n} z_{n}-p\right\|^{2}+\left(1-\alpha_{n}\right)\left\|T_{n} z_{n}-p\right\|^{2} \\
\leq & \alpha_{n}\left\|u+\gamma f\left(x_{n}\right)+(I-\bar{A}) T_{n} z_{n}-p\right\|^{2}+\left(1-\alpha_{n}\right)\left\|z_{n}-p\right\|^{2} \\
\leq & \alpha_{n}\left\|u+\gamma f\left(x_{n}\right)+(I-\bar{A}) T_{n} z_{n}-p\right\|^{2} \\
& +\left(1-\alpha_{n}\right)\left\{\left\|x_{n}-p\right\|^{2}-\left\|u_{n}-z_{n}\right\|^{2}\right. \\
& \left.+2 \lambda_{n}\left\langle u_{n}-z_{n}, F u_{n}-F p\right\rangle-\lambda_{n}^{2}\left\|F u_{n}-F p\right\|^{2}\right\} \\
\leq & \alpha_{n}\left\|u+\gamma f\left(x_{n}\right)+(I-\bar{A}) T_{n} z_{n}-p\right\|^{2}+\left\|x_{n}-p\right\|^{2} \\
& -\left(1-\alpha_{n}\right)\left\|u_{n}-z_{n}\right\|^{2}+2 \lambda_{n}\left(1-\alpha_{n}\right)\left\langle u_{n}-z_{n}, F u_{n}-F p\right\rangle \\
& -\lambda_{n}^{2}\left\|F u_{n}-F p\right\|^{2} .
\end{aligned}
$$

Hence, we obtain

$$
\begin{aligned}
\left\|x_{n+1}-p\right\|^{2} \leq & \left\|y_{n}-p\right\|^{2} \\
\leq & \alpha_{n}\left\|u+\gamma f\left(x_{n}\right)+(I-\bar{A}) T_{n} z_{n}-p\right\|^{2}+\left\|x_{n}-p\right\|^{2} \\
& -\left(1-\alpha_{n}\right)\left\|u_{n}-z_{n}\right\|^{2}+2 \lambda_{n}\left(1-\alpha_{n}\right)\left\langle u_{n}-z_{n}, F u_{n}-F p\right\rangle \\
& -\lambda_{n}^{2}\left\|F u_{n}-F p\right\|^{2}
\end{aligned}
$$

which implies that

$$
\begin{aligned}
& \left(1-\alpha_{n}\right)\left\|u_{n}-z_{n}\right\|^{2} \\
\leq & \alpha_{n}\left\|u+\gamma f\left(x_{n}\right)+(I-\bar{A}) T_{n} z_{n}-p\right\|^{2}+\left\|x_{n}-p\right\|^{2}-\left\|x_{n+1}-p\right\|^{2} \\
& +2 \lambda_{n}\left(1-\alpha_{n}\right)\left\|u_{n}-z_{n}\right\|\left\|F u_{n}-F p\right\| \\
\leq & \alpha_{n}\left\|u+\gamma f\left(x_{n}\right)+(I-\bar{A}) T_{n} z_{n}-p\right\|^{2}+\left\|x_{n+1}-x_{n}\right\|\left(\left\|x_{n}-p\right\|+\left\|x_{n+1}-p\right\|\right) \\
& +2 \lambda_{n}\left(1-\alpha_{n}\right)\left\|u_{n}-z_{n}\right\|\left\|F u_{n}-F p\right\| .
\end{aligned}
$$

From $\left\|x_{n+1}-x_{n}\right\| \rightarrow 0, \alpha_{n} \rightarrow 0$ and $\left\|F u_{n}-F p\right\| \rightarrow 0$ as $n \rightarrow \infty$, it follows that

$$
\lim _{n \rightarrow \infty}\left\|u_{n}-z_{n}\right\|=0
$$

Since $\left\|T_{n} z_{n}-z_{n}\right\| \leq\left\|T_{n} z_{n}-x_{n}\right\|+\left\|x_{n}-u_{n}\right\|+\left\|u_{n}-z_{n}\right\|$, from Step 3, Step 4, and (3.28), we conclude that

$$
\lim _{n \rightarrow \infty}\left\|T_{n} z_{n}-z_{n}\right\|=0 .
$$

We notice that by the assumption on $T$, (3.29) and Lemma 2.5,

$$
\begin{aligned}
\lim _{n \rightarrow \infty}\left\|T z_{n}-z_{n}\right\| & \leq \lim _{n \rightarrow \infty}\left(\left\|T z_{n}-T_{n} z_{n}\right\|+\left\|T_{n} z_{n}-z_{n}\right\|\right) \\
& \leq \lim _{n \rightarrow \infty} \sup \left\{\left\|T y-T_{n} y\right\|: y \in C\right\}+\lim _{n \rightarrow \infty}\left\|T_{n} z_{n}-z_{n}\right\|=0 .
\end{aligned}
$$

Step 6: We show that

$$
\limsup _{n \rightarrow \infty}\left\langle u+(\gamma f-(I+\mu A)) q, y_{n}-q\right\rangle=\limsup _{n \rightarrow \infty}\left\langle u+(\gamma f-\bar{A}) q, y_{n}-q\right\rangle \leq 0,
$$

where $q$ is a solution of the optimization problem (OP1). To this end, first we prove that

$$
\limsup _{n \rightarrow \infty}\left\langle u+(\gamma f-\bar{A}) q, T z_{n}-q\right\rangle \leq 0
$$


Since $\left\{z_{n}\right\}$ is bounded, we can choose a subsequence $\left\{z_{n_{i}}\right\}$ of $\left\{z_{n}\right\}$ such that

$$
\limsup _{n \rightarrow \infty}\left\langle u+(\gamma f-\bar{A}) q, T z_{n}-q\right\rangle=\lim _{i \rightarrow \infty}\left\langle u+(\gamma f-\bar{A}) q, T z_{n_{i}}-q\right\rangle .
$$

Without loss of generality, we may assume that $\left\{z_{n_{i}}\right\}$ converges weakly to $z \in C$. From $\left\|T z_{n}-z_{n}\right\| \rightarrow 0$ in (3.30), it follows that $T z_{n_{i}} \rightarrow z$.

Now, we will show that $z \in \Omega_{1}$. First, we show that $z \in \bigcap_{n=1}^{\infty} F\left(T_{n}\right)=F(T)$. Assume that $z \notin F(T)$. Since $z_{n_{i}} \rightarrow z$ and $T z \neq z$, by the Opial condition, we obtain

$$
\begin{aligned}
\liminf _{i \rightarrow \infty}\left\|z_{n_{i}}-z\right\| & <\liminf _{i \rightarrow \infty}\left\|z_{n_{i}}-T z\right\| \\
& \leq \liminf _{i \rightarrow \infty}\left(\left\|z_{n_{i}}-T z_{n_{i}}\right\|+\left\|T z_{n_{i}}-T z\right\|\right) \\
& \leq \liminf _{i \rightarrow \infty}\left\|z_{n_{i}}-z\right\|,
\end{aligned}
$$

which is a contradiction. Thus we have $z \in \bigcap_{n=1}^{\infty} F\left(T_{n}\right)=F(T)$.

Next, we prove that $z \in V I(C, F)$. Let

$$
Q v= \begin{cases}F v+N_{C} v, & v \in C \\ \emptyset, & v \notin C,\end{cases}
$$

where $N_{C} v$ is normal cone to $C$ at $v$. We already know that, in this case, the mapping $Q$ is maximal monotone, and $0 \in Q v$ if and only if $v \in V I(C, F)$. Let $(v, w) \in G(Q)$. Since $w-F v \in N_{C} v$ and $z_{n} \in C$, we have

$$
\left\langle v-z_{n}, w-F v\right\rangle \geq 0 .
$$

On the other hand, from $z_{n}=P_{C}\left(u_{n}-\lambda_{n} F u_{n}\right)$, we have

$$
\left\langle v-z_{n}, z_{n}-\left(u_{n}-\lambda_{n} F u_{n}\right)\right\rangle \geq 0,
$$

that is,

$$
\left\langle v-z_{n}, \frac{z_{n}-u_{n}}{\lambda_{n}}+F u_{n}\right\rangle \geq 0 .
$$

Thus, we obtain

$$
\begin{aligned}
\left\langle v-z_{n_{i}}, w\right\rangle \geq & \left\langle v-z_{n_{i}}, F v\right\rangle \\
\geq & \left\langle v-z_{n_{i}}, F v\right\rangle-\left\langle v-z_{n_{i}}, \frac{z_{n_{i}}-u_{n_{i}}}{\lambda_{n_{i}}}+F u_{n_{i}}\right\rangle \\
= & \left\langle v-z_{n_{i}}, F v-F z_{n_{i}}\right\rangle+\left\langle v-z_{n_{i}}, F z_{n_{i}}-F u_{n_{i}}\right\rangle \\
& \quad-\left\langle v-z_{n_{i}}, \frac{z_{n_{i}}-u_{n_{i}}}{\lambda_{n_{i}}}\right\rangle \\
& \quad\left\langle v-z_{n_{i}}, F z_{n_{i}}-F u_{n_{i}}\right\rangle-\left\langle v-z_{n_{i}} \frac{z_{n_{i}}-u_{n_{i}}}{\lambda_{n_{i}}}\right\rangle .
\end{aligned}
$$

Since $\left\|z_{n}-u_{n}\right\| \rightarrow 0$ in (3.28) and $F$ is $\alpha$-inverse-strongly monotone, it follows from (3.32) that

$$
\langle v-z, w\rangle \geq 0, \quad \text { as } i \rightarrow \infty .
$$

Since $Q$ is maximal monotone, we have $z \in Q^{-1} 0$ and hence $z \in V I(C, F)$. 
Finally, we show that $z \in G M E P(\Theta, \phi, B)$. By $u_{n}=S_{r_{n}}\left(x_{n}-r_{n} B x_{n}\right)$; we know that

$$
\Theta\left(u_{n}, y\right)+\left\langle B x_{n}, y-u_{n}\right\rangle+\varphi(y)-\varphi\left(u_{n}\right)+\frac{1}{r_{n}}\left\langle y-u_{n}, u_{n}-x_{n}\right\rangle \geq 0, \quad \forall y \in C .
$$

It follows from (A2) that

$$
\left\langle B x_{n}, y-u_{n}\right\rangle+\varphi(y)-\varphi\left(u_{n}\right)+\frac{1}{r_{n}}\left\langle y-u_{n}, u_{n}-x_{n}\right\rangle \geq \Theta\left(y, u_{n}\right), \quad \forall y \in C .
$$

Hence

$$
\left\langle B x_{n_{i}}, y-u_{n_{i}}\right\rangle+\left(\varphi(y)-\left(\varphi\left(u_{n_{i}}\right)+\frac{1}{r_{n_{i}}}\left\langle y-u_{n_{i}}, u_{n_{i}}-x_{n_{i}}\right\rangle \geq \Theta\left(y, u_{n_{i}}\right), \quad \forall y \in \text { Q } 3.33\right)\right.
$$

For $t$ with $0<t \leq 1$ and $y \in C$, let $y_{t}=t y+(1-t) z$. Since $y \in C$ and $z \in C$, we have $y_{t} \in C$ and hence $\Theta\left(y_{t}, z\right) \leq 0$. Hence, from (3.33), we have

$$
\begin{aligned}
\left\langle y_{t}-u_{n_{i}}, B y_{t}\right\rangle \geq & \left\langle y_{t}-u_{n_{i}}, B y_{t}\right\rangle-\varphi\left(y_{t}\right)+\varphi\left(u_{n_{i}}\right)-\left\langle y_{t}-u_{n_{i}}, B x_{n_{i}}\right\rangle \\
& -\left\langle y_{t}-u_{n_{i}}, \frac{u_{n_{i}}-x_{n_{i}}}{r_{n_{i}}}\right\rangle+\Theta\left(y_{t}, u_{n_{i}}\right) \\
= & \left\langle y_{t}-u_{n_{i}}, B y_{t}-B u_{n_{i}}\right\rangle+\left\langle y_{t}-u_{n_{i}}, B u_{n_{i}}-B x_{n_{i}}\right\rangle \\
& -\varphi\left(y_{t}\right)+\varphi\left(u_{n_{i}}\right)-\left\langle y_{t}-u_{n_{i}}, \frac{u_{n_{i}}-x_{n_{i}}}{r_{n_{i}}}\right\rangle+\Theta\left(y_{t}, u_{n_{i}}\right)
\end{aligned}
$$

Since $\left\|u_{n_{i}}-x_{n_{i}}\right\| \rightarrow 0$ from Step 3 , we have $\left\|B u_{n_{i}}-B x_{n_{i}}\right\| \rightarrow 0$ and $\frac{u_{n_{i}}-x_{n_{i}}}{r_{n_{i}}} \rightarrow 0$. Also by $\left\|u_{n}-z_{n}\right\| \rightarrow 0$ in (3.28), we have $u_{n_{i}} \rightarrow z$. Moreover, from the inverse-strongly monotonicity of $B$, we have $\left\langle y_{t}-u_{n_{i}}, B y_{t}-B u_{n_{i}}\right\rangle \geq 0$. Hence, from (A4) and the weak lower semicontinuity of $\phi$, if follows that

$$
\left\langle y_{t}-z, B y_{t}\right\rangle \geq-\varphi\left(y_{t}\right)+\varphi(z)+\Theta\left(y_{t}, z\right) \text { as } i \rightarrow \infty \text {. }
$$

From (A1), (A4), and (3.34), we also obtain

$$
\begin{aligned}
0 & =\Theta\left(y_{t}, y_{t}\right)+\varphi\left(y_{t}\right)-\varphi\left(y_{t}\right) \\
& \leq t \Theta\left(y_{t}, y\right)+(1-t) \Theta\left(y_{t}, z\right)+t \varphi\left(y_{t}\right)+(1-t) \varphi(z)-\varphi\left(y_{t}\right) \\
& \leq t\left[\Theta\left(y_{t}, y\right)+\varphi(\gamma)-\varphi\left(y_{t}\right)\right]+(1-t)\left\langle y_{t}-z, B y_{t}\right\rangle \\
& =t\left[\Theta\left(y_{t}, y\right)+\varphi(\gamma)-\varphi\left(y_{t}\right)\right]+(1-t) t\left\langle y-z, B y_{t}\right\rangle,
\end{aligned}
$$

and hence

$$
0 \leq \Theta\left(y_{t}, y\right)+\varphi(y)-\varphi\left(y_{t}\right)+(1-t)\left\langle y-z, B y_{t}\right\rangle .
$$

Letting $t \rightarrow 0$ in (3.35), we have for each $y \in C$

$$
\Theta(z, y)+\langle B z, y-z\rangle+\varphi(\gamma)-\varphi(z) \geq 0
$$

This implies that $z \in G M E P(\Theta, \phi, B)$. Therefore $z \in \Omega_{1}$.

Now, from Lemma 2.6 and (3.31), we obtain

$$
\begin{aligned}
\limsup _{n \rightarrow \infty}\left\langle u+(\gamma f-\bar{A}) q, T z_{n}-q\right\rangle & =\lim _{i \rightarrow \infty}\left\langle u+(\gamma f-\bar{A}) q, T z_{n_{i}}-q\right\rangle \\
& =\langle u+(\gamma f-\bar{A}) q, z-q\rangle \\
& \leq 0 .
\end{aligned}
$$


Since $\lim _{n \rightarrow \infty}|| x_{n}-T z_{n}|| \leq\left\|x_{n}-T_{n} z_{n}||+\right\| T_{n} z_{n}-T z_{n} \| \rightarrow 0$, from Step 4 and Lemma 2.5, and from (3.36), we conclude that

$$
\begin{aligned}
& \limsup _{n \rightarrow \infty}\left\langle u+(\gamma f-\bar{A}) q, y_{n}-q\right\rangle \\
\leq & \limsup _{n \rightarrow \infty}\left\langle u+(\gamma f-\bar{A}) q, y_{n}-T z_{n}\right\rangle+\limsup _{n \rightarrow \infty}\left\langle u+(\gamma f-\bar{A}) q, T z_{n}-q\right\rangle \\
\leq & \limsup _{n \rightarrow \infty}\|u+(\gamma f-\bar{A}) q\|\left\|y_{n}-T z_{n}\right\|+\limsup _{n \rightarrow \infty}\left\langle u+(\gamma f-\bar{A}) q, T z_{n}-q\right\rangle \\
\leq & 0 .
\end{aligned}
$$

Step 7: We show that $\lim _{n \rightarrow \infty}\left\|x_{n}-q\right\|=0$ and $\lim _{n \rightarrow \infty}\left\|u_{n}-q\right\|=0$, where $q$ is a solution of the optimization problem (OP1). Indeed, from (IS) and Lemma 2.4, we have

$$
\begin{aligned}
&\left\|x_{n+1}-q\right\|^{2} \leq\left\|y_{n}-q\right\|^{2} \\
& \leq\left\|\left(I-\alpha_{n} \bar{A}\right)\left(T_{n} z_{n}-q\right)\right\|^{2}+2 \alpha_{n}\left\langle u+\gamma f\left(x_{n}\right)-\bar{A} q, y_{n}-q\right\rangle \\
& \leq\left(1-(1+\mu) \bar{\gamma} \alpha_{n}\right)^{2}\left\|z_{n}-q\right\|^{2}+2 \alpha_{n} \gamma\left\langle f\left(x_{n}\right)-f(q), y_{n}-q\right\rangle \\
&\left.+2 \alpha_{n}\left\langle u+\gamma f(q)-\bar{A} q, y_{n}-q\right\rangle\right) \\
& \leq\left(1-(1+\mu) \bar{\gamma} \alpha_{n}\right)^{2}\left\|x_{n}-q\right\|^{2}+2 \alpha_{n} \gamma k\left\|x_{n}-q\right\|\left\|y_{n}-q\right\| \\
&+2 \alpha_{n}\left\langle u+(\gamma f-\bar{A}) q, y_{n}-q\right\rangle \\
& \leq\left(1-(1+\mu) \bar{\gamma} \alpha_{n}\right)^{2}\left\|x_{n}-q\right\|^{2}+2 \alpha_{n} \gamma k\left\|x_{n}-q\right\|\left(\left\|y_{n}-x_{n}\right\|+\left\|x_{n}-q\right\|\right) \\
&+2 \alpha_{n}\left\langle u+(\gamma f-\bar{A}) q, y_{n}-q\right\rangle \\
&=\left(1-2((1+\mu) \bar{\gamma}-\gamma k) \alpha_{n}\right)\left\|x_{n}-q\right\|^{2} \\
&+\alpha_{n}^{2}((1+\mu) \bar{\gamma})^{2}\left\|x_{n}-q\right\|^{2}+2 \alpha_{n} \gamma k\left\|x_{n}-q\right\|\left\|y_{n}-x_{n}\right\| \\
&+2 \alpha_{n}\left\langle u+(\gamma f-\bar{A}) q, y_{n}-q\right\rangle,
\end{aligned}
$$

that is,

$$
\begin{aligned}
\left\|x_{n+1}-q\right\|^{2} \leq & \left(1-2((1+\mu) \bar{\gamma}-\gamma k) \alpha_{n}\right)\left\|x_{n}-q\right\|^{2}+\alpha_{n}^{2}((1+\mu) \bar{\gamma})^{2} M_{6} \\
& +2 \alpha_{n} \gamma k\left\|y_{n}-x_{n}\right\| M_{6}+2 \alpha_{n}\left\langle u+(\gamma f-\bar{A}) q, y_{n}-q\right\rangle \\
= & \left(1-\overline{\alpha_{n}}\right)\left\|x_{n}-q\right\|^{2}+\overline{\beta_{n}}
\end{aligned}
$$

where $M_{6}=\sup \left\{\left\|x_{n}-q\right\|: n \geq 1\right\}, \overline{\alpha_{n}}=2((1+\mu) \bar{\gamma}-\gamma k) \alpha_{n}$ and

$$
\overline{\beta_{n}}=\alpha_{n}\left[\alpha_{n}((1+\mu) \bar{\gamma})^{2} M_{6}^{2}+2 \gamma k\left\|\gamma_{n}-x_{n}\right\| M_{6}+2\left\langle u+(\gamma f-\bar{A}) q, \gamma_{n}-q\right\rangle\right] \text {. }
$$

From the condition (C1), $\left\|y_{n}-x_{n}\right\| \rightarrow 0$ in Step 4, and Step 6, it is easily seen that $\sum_{n=1}^{\infty} \overline{\alpha_{n}}=\infty, \sum_{n=1}^{\infty} \overline{\alpha_{n}}=\infty$, and $\lim \sup _{n \rightarrow \infty} \frac{\overline{\beta_{n}}}{\overline{\alpha_{n}}} \leq 0$. Hence, by Lemma 2.3, we conclude $x_{n} \rightarrow q$ as $n \rightarrow \infty$. Moreover, from Step 3, we obtain that $u_{n} \rightarrow q$ as $n \rightarrow \infty$. This completes the proof. $\square$

Corollary 3.2 Let H, C, $\Theta, \phi, F, A, f, \mu, \gamma, \bar{\gamma}$ and $k$ be as in Theorem 3.1. Let $\left\{T_{n}\right\}$ be a sequence of nonexpansive mappings of $C$ into itself such that $\Omega_{2}:=\bigcap_{n=1}^{\infty} F\left(T_{n}\right) \cap \operatorname{VI}(C, F) \cap \operatorname{MEP}(\Theta, \varphi) \neq \emptyset$. Assume that either (B1) or (B2) holds. Let $u \in C$ and let $\left\{x_{n}\right\}$ and $\left\{u_{n}\right\}$ be sequences generated by $x_{1} \in C$ and

$$
\left\{\begin{array}{l}
\Theta\left(u_{n}, \gamma\right)+\varphi(y)-\varphi\left(u_{n}\right)+\frac{1}{r_{n}}\left\langle y-u_{n}, u_{n}-x_{n}\right\rangle \geq 0, \quad \forall y \in C, \\
y_{n}=\alpha_{n}\left(u+\gamma f\left(x_{n}\right)\right)+\left(I-\alpha_{n}(I+\mu A)\right) T_{n} P_{C}\left(u_{n}-\lambda_{n} F u_{n}\right), \\
x_{n+1}=\left(1-\beta_{n}\right) y_{n}+\beta_{n} T_{n} P_{C}\left(y_{n}-\lambda_{n} F y_{n}\right), \quad n \geq 1,
\end{array}\right.
$$


where $\left\{\alpha_{n}\right\},\left\{\beta_{n}\right\} \subset[0,1], \lambda_{n} \in[a, b] \subset(0,2 \alpha)$, and $r_{n} \in[c, d] \subset(0,2 \beta)$. Let $\left\{\alpha_{n}\right\}$, $\left\{\beta_{n}\right\},\left\{\lambda_{n}\right\}$, and $\left\{r_{n}\right\}$ satisfy the conditions (C1), (C2), and (C3) in Theorem 3.1. Suppose that $\sum_{n=1}^{\infty} \sup \left\{\left\|T_{n+1} z-T_{n} z\right\|: z \in D\right\}<\infty$ for any bounded subset $D$ of $C$. Let $T$ be a mapping of $C$ into itself defined by $T z=\lim _{n \rightarrow \infty} T_{n} z$ for all $z \in C$ and suppose that $F(T)=\bigcap_{n=1}^{\infty} F\left(T_{n}\right)$. Then $\left\{x_{n}\right\}$ and $\left\{u_{n}\right\}$ converge strongly to $q \in \Omega_{2}$, which is a solution of the optimization problem:

$$
\min _{x \in \Omega_{2}} \frac{\mu}{2}\langle A x, x\rangle+\frac{1}{2}\|x-u\|^{2}-h(x), \quad(\mathrm{OP} 2)
$$

where $h$ is a potential function for $\gamma f$.

Proof Putting $B=0$ in Theorem 3.1, we obtain the required result.

As direct consequences of Theorem 3.1, we can also obtain the following new strong convergence theorems for the problem (1.1) and fixed point problem.

Corollary 3.3 Let $\mathrm{H}, \mathrm{C}, \Theta, \phi, \mathrm{B}, \mathrm{A}, \mathrm{f}, \mu, \gamma, \bar{\gamma}$, and $k$ be as in Theorem 3.1. Let $T$ be a nonexpansive mapping of $C$ into itself such that $\Omega_{3}:=F(T) \cap G M E P(\Theta, \phi, B) \neq \varnothing$. Assume that either (B1) or (B2) holds. Let $u \in C$ and let $\left\{x_{n}\right\}$ and $\left\{u_{n}\right\}$ be sequences generated by $x_{1} \in C$ and

$$
\left\{\begin{array}{l}
\Theta\left(u_{n}, \gamma\right)+\left\langle B x_{n}, y-u_{n}\right\rangle+\varphi(y)-\varphi\left(u_{n}\right)+\frac{1}{r_{n}}\left\langle y-u_{n}, u_{n}-x_{n}\right\rangle \geq 0, \quad \forall y \in C, \\
x_{n+1}=\alpha_{n}\left(u+\gamma f\left(x_{n}\right)\right)+\left(I-\alpha_{n}(I+\mu A)\right) T u_{n}, \quad n \geq 1,
\end{array}\right.
$$

where $\left\{\alpha_{n}\right\} \subset[0,1]$, and $r_{n} \in[c, d] \subset(0 ; 2 \beta)$. Let $\left\{\alpha_{n}\right\}$ and $\left\{r_{n}\right\}$ satisfy the following conditions:

(i) $\alpha_{n} \rightarrow 0(n \rightarrow \infty) ; \sum_{n=1}^{\infty} \alpha_{n}=\infty$;

(ii) $\sum_{n=1}^{\infty}\left|\alpha_{n+1}-\alpha_{n}\right|<\infty ; \sum_{n=1}^{\infty}\left|r_{n+1}-r_{n}\right|<\infty$

Then $\left\{x_{n}\right\}$ and $\left\{u_{n}\right\}$ converge strongly to $q \in \Omega_{3}$, which is a solution of the optimization problem:

$$
\min _{x \in \Omega_{3}} \frac{\mu}{2}\langle A x, x\rangle+\frac{1}{2}\|x-u\|^{2}-h(x),
$$

where $h$ is a potential function for $\gamma f$.

Proof Putting $F=0, \beta_{n}=0$, and $T_{n}=T$ for $n \geq 1$ in Theorem 3.1, we obtain the required result. $\square$

A mapping $S: C \rightarrow C$ is called strictly pseudocontractive if there exists $r$ with $0 \leq r$ $<1$ such that

$$
\|S x-S y\|^{2} \leq\|x-y\|^{2}+r\|(I-S) x-(I-S) y\|^{2} \text { for all } x, y \in C .
$$

Such a mapping $S$ is called $r$-strictly pseudocontractive. Putting $F=I-S$, we know that

$$
\langle x-y, F x-F y\rangle \geq \frac{1-r}{2}\|F x-F y\|^{2} \text { for all } x, y \in C ;
$$

see, for instance, [12,29]. Hence, we have the following result.

Corollary 3.4 Let $H, C, \Theta, \phi, B, A, f, \mu, \gamma, \bar{\gamma}$, and $k$ be as in Theorem 3.1. Let $S$ be a $r$-strictly pseudocontractive mappings of $C$ into itself. Let $T$ be a nonexpansive mapping of $C$ into itself such that $\Omega_{4}:=F(T) \cap F(S) \cap G M E P(\Theta, \phi, B) \neq \varnothing$. Assume that either 
(B1) or (B2) holds. Let $u \in C$ and let $\left\{x_{n}\right\}$ and $\left\{u_{n}\right\}$ be sequences generated by $x_{1} \in C$ and

$$
\left\{\begin{array}{l}
\Theta\left(u_{n}, y\right)+\left\langle B x_{n}, y-u_{n}\right\rangle+\varphi(y)-\varphi\left(u_{n}\right)+\frac{1}{r_{n}}\left\langle y-u_{n}, u_{n}-x_{n}\right\rangle \geq 0, \quad \forall y \in C, \\
x_{n+1}=\alpha_{n}\left(u+\gamma f\left(x_{n}\right)\right)+\left(I-\alpha_{n}(I+\mu A)\right) T\left(u_{n}-\lambda_{n}\left(u_{n}-S u_{n}\right)\right), \quad n \geq 1,
\end{array}\right.
$$

where $\left\{\alpha_{n}\right\},\left\{\lambda_{n}\right\} \subset[0,1], \lambda_{n} \in[a, b] \subset(0,1-r)$, and $r_{n} \in[c, d] \subset(0,2 \beta)$. Let $\left\{\alpha_{n}\right\}$, $\left\{\lambda_{n}\right\}$, and $\left\{r_{n}\right\}$ satisfy the following conditions:

(i) $\alpha_{n} \rightarrow 0(n \rightarrow \infty) ; \sum_{n=1}^{\infty} \alpha_{n}=\infty$;

(ii) $\sum_{n=1}^{\infty}\left|\alpha_{n+1}-\alpha_{n}\right|<\infty ; \sum_{n=1}^{\infty}\left|\lambda_{n+1}-\lambda_{n}\right|<\infty ; \sum_{n=1}^{\infty}\left|r_{n+1}-r_{n}\right|<\infty$.

Then $\left\{x_{n}\right\}$ and $\left\{u_{n}\right\}$ converge strongly to $q \in \Omega_{4}$, which is a solution of the optimization problem

$$
\min _{x \in \Omega_{4}} \frac{\mu}{2}\langle A x, x\rangle+\frac{1}{2}\|x-u\|^{2}-h(x),
$$

where $h$ is a potential function for $\gamma f$.

Proof Putting $F=I-S, \beta_{n}=0$, and $T_{n}=T$ for $n \geq 1$, from Theorem 3.1, we obtain the required result. $\square$

\section{Remark 3.1}

(1) Theorem 3.1, Corollary 3.2, Corollary 3.3, and Corollary 3.4 improve and develop the corresponding results, which were obtained recently by many authors in various references, for example, see [2,3,7-9,11-18,24,30]. In particular, we mention that the iterative scheme (3.1) in Theorem 3.1 of [24] is not well defined without the assumption $C \pm C \subset C$ on a nonempty closed convex subset $C$ of $H$.

(2) The condition $\sum_{n=1}^{\infty}\left|\alpha_{n+1}-\alpha_{n}\right|<\infty$ imposed on $\left\{\alpha_{n}\right\}$ can be replaced by the perturbed control condition $\left|\alpha_{n+1}-\alpha_{n}\right|<o\left(\alpha_{n+1}\right)+\sigma_{n}, \sum_{n=1}^{\infty} \sigma_{n}<\infty$ or the condition $\alpha_{n} \in(0,1], n \geq 1$, and $\lim _{n \rightarrow \infty} \alpha_{n} / \alpha_{n+1}=1$.

(3) Some special cases of the generalized mixed equilibrium problem (1.1) are known as follows:

(i) If $\phi=0$, then the problem (1.1) reduced the following generalized equilibrium problem $(G E P)$ of finding $x \in C$ such that

$\Theta(x, y)+\langle B x, y-x\rangle \geq 0, \quad \forall y \in C$,

which was studied by Takahashi and Takahashi [30].

(ii) If $\Theta(x, y)=0$ for all $x, y \in C$, then the problem (1.1) reduces the following generalized variational inequality problem $(G V I)$ of finding $x \in C$ such that

$\langle B x, y-x\rangle+\varphi(y)-\varphi(x) \geq 0, \quad \forall y \in C$.

(iii) If $B=0$ and $\Theta(x, y)=0$ for all $x, y \in C$, then the problem (1.1) reduces the following minimization problem of finding $x \in C$ such that 


$$
\varphi(y)-\varphi(x) \geq 0, \quad \forall y \in C .
$$

Applying Theorem 3.1 together with the one in the following assumptions instead of (B1), we can also establish the new corresponding results for the above mentioned problems:

(B2) $C$ is a bounded set;

(B3) For each $x \in H$ and $r>0$, there exist a bounded subset $D_{x} \subseteq C$ and $y_{x} \in C$ such that for any $z \in C \backslash D_{x}$,

$$
\varphi\left(\gamma_{x}\right)-\varphi(z)+\frac{1}{r}\left\langle y_{x}-z, z-x\right\rangle<0 ;
$$

(B4) For each $x \in H$ and $r>0$, there exist a bounded subset $D_{x} \subseteq C$ and $y_{x} \in C$ such that for any $z \in C \backslash D_{x}$,

$$
\Theta\left(z, y_{x}\right)+\frac{1}{r}\left\langle y_{x}-z, z-x\right\rangle<0 .
$$

\section{Acknowledgements}

The author would like to thank the anonymous referees for their valuable comments and suggestions, which improved the presentation of this manuscript. This research was supported by the Basic Science Research Program through the National Research Foundation of Korea (NRF) funded by the Ministry of Education, Science and Technology (2010-0017007).

\section{Competing interests}

The author declares that they have no competing interests.

Received: 21 February 2011 Accepted: 8 September 2011 Published: 8 September 2011

\section{References}

1. Peng, J-W, Yao, J-C: A new hybrid-extra gradient method for generalized mixed equilibrium problems, fixed point problems and variational inequality problems. Taiwan J Math. 12, 1401-1432 (2008)

2. Ceng, L-C, Yao, J-C: A hybrid iterative scheme for mixed equilibrium problems and fixed point problems. J Comput Appl Math. 214, 186-201 (2008). doi:10.1016/j.cam.2007.02.022

3. Yao, YH, Noor, MA, Zainab, S, Liou, Y-C: Mixed equilibrium problems and optimization problems. J Math Anal Appl. 354, 319-329 (2009). doi:10.1016/j.jmaa.2008.12.055

4. Blum, E, Oettli, W: From optimization and variational inequalities to equilibrium problems. Math Student. 63, 123-145 (1994)

5. Combettes, Pl, Hirstoaga, SA: Equilibrium programming in Hilbert spaces. J Nonlinear Convex Anal. 6, 117-136 (2005)

6. Flam, SD, Antipin, AS: Equilibrium programming using proximal-like algorithm. Math Program. 78, 29-41 (1997)

7. Chang, S-S, Joseph Lee, HW, Chan, CK: A new method for solving equilibrium problem, fixed point problem and variational inequality problem with application to optimization. Nonlinear Anal. 70, 3307-3319 (2009). doi:10.1016/j. na.2008.04.035

8. Colao, V, Marino, G, Xu, HK: An iterative method for finding common solutions of equilibrium problems and fixed point problems. J Math Anal Appl. 344, 340-352 (2008). doi:10.1016/j.jmaa.2008.02.041

9. Jung, JS: Strong convergence of composite iterative methods for equilibrium problems and fixed point problems. Appl Math Comput. 213, 498-505 (2009). doi:10.1016/j.amc.2009.03.048

10. Plubtieng, S, Punpaeng, R: A general iterative method for equilibrium problems and fixed point problems in Hilbert spaces. J Math Anal Appl. 336, 445-468 (2007)

11. Plubtieng, S, Punpaeng, R: A new iterative method for equilibrium problems and fixed point problems of nonlinear mappings and monotone mappings. Appl Math Comput. 197, 548-558 (2008). doi:10.1016/j.amc.2007.07.075

12. Tada, A, Takahashi, W: Weak and strong convergence theorems for a nonexpansive mapping and an equilibrium problem. J Optim Theory Appl. 133, 359-370 (2007). doi:10.1007/s10957-007-9187-z

13. Takahashi, A, Takahashi, W: Viscosity approximation methods for equilibrium problems and fixed point problems in Hilbert spaces. J Math Anal Appl. 331, 506-515 (2007). doi:10.1016/j.jmaa.2006.08.036

14. Wang, S, Hu, C, Chai, G: Strong convergence of a new composite iterative method for equilibrium problems and fixed point problems. Appl Math Comput. 215, 3891-3898 (2010). doi:10.1016/j.amc.2009.11.036

15. Yao, Y, Liou, YC, Yao, J-C: Convergence theorem for equilibrium problems and fixed point problems of infinite family of nonexpansive mappings. Fixed Point Theory Appl 2007, 12 (2007). Article ID 64363

16. Yao, YH, Noor, MA, Liou, Y-C: On iterative methods for equilibrium problems. Nonlinear Anal. 70, 497-509 (2009). doi:10.1016/j.na.2007.12.021

17. Su, Y, Shang, M, Qin, $X:$ An iterative method of solution for equilibrium problems and optimization problems. Nonlinear Anal. 69, 2709-2719 (2008). doi:10.1016/j.na.2007.08.045 
18. Yao, Y, Cho, YJ, Chen, R: An iterative algorithm for solving fixed point problems, variational inequality problems and mixed equilibrium problems. Nonlinear Anal. 71, 3363-3373 (2009). doi:10.1016/.jna.2009.01.236

19. Bauschke, HH, Borwein, JM: On projection algorithms for solving convex feasibility problems. SIAM Rev. 38, 367-426 (1997)

20. Combettes, PL: Hilbertian convex feasibility problem: convergence of projection methods. Appl Math Optim. 35, 311-330 (1997)

21. Deutsch, F, Yamada, I: Minimizing certain convex functions over the intersection of the fixed point sets of nonexpansive mappings. Numer Funct Anal Optim. 19, 33-56 (1998)

22. Jung, JS: Iterative algorithms with some control conditions for quadratic optimizations. Panamer Math J. 16(4), 13-25 (2006)

23. Xu, HK: An iterative approach to quadratic optimization. J Optim Theory Appl. 116, 659-678 (203)

24. Jaiboon, C, Kumam, P: A general iterative method for addressing mixed equilibrium problems and optimizations. Nonlinear Anal. 73, 1180-1202 (2010). doi:10.1016/..na.2010.04.041

25. Takahashi, W, Toyoda, M: Weak convergence theorems for nonexpansive mappings and monotone mappings. J Optim Theory Appl. 118(2), 417-428 (2003). doi, 10.1023/A:1025407607560

26. Rockafellar, RT: On the maximality of sums of nonlinear monotone operators. Trans Am Math Soc. 149, 75-88 (1970). doi:10.1090/S0002-9947-1970-0282272-5

27. Rockafellar, RT: Monotone operators and the proximal point theorems. SIAM J Control Optim. 14, 877-898 (1976). doi:10.1137/0314056

28. Aoyama, K, Kimura, Y, Takahashi, W, Toyoda, M: Approximation of common fixed points of a countable family of nonexpansive mappings in a Banach space. Nonlinear Anal. 67, 2350-2360 (2007). doi:10.1016/.jna.2006.08.032

29. Jung, JS: A new iteration method for nonexpansive mappings and monotone mappings in Hilbert spaces. I Inequal Appl 2010, 16 (2010). Article ID 251761

30. Takahashi, S, Takahashi, W: Strong convergence theorem for a generalized equilibrium problem and a nonexpansive mapping in a Hilbert space. Nonlinear Anal. 69, 1025-1033 (2008). doi:10.1016/j.na.2008.02.042

doi:10.1186/1029-242X-2011-51

Cite this article as: Jung: A general composite iterative method for generalized mixed equilibrium problems, variational inequality problems and optimization problems. Journal of Inequalities and Applications 2011 2011:51.

\section{Submit your manuscript to a SpringerOpen ${ }^{\circ}$ journal and benefit from:}

- Convenient online submission

- Rigorous peer review

- Immediate publication on acceptance

- Open access: articles freely available online

- High visibility within the field

- Retaining the copyright to your article

Submit your next manuscript at $\gg$ springeropen.com 